\title{
Analysis of BMP3 Variants In The Causality of Ocular Coloboma
}

\section{Sabrina Fox}

University of Alberta https://orcid.org/0000-0003-1534-2006

\section{Sonya A. Widen}

University of Alberta

Mika Asai-Coakwell

University of Saskatchewan

\section{Serhiy Havrylov}

University of Alberta

\section{Matthew Benson}

University of Alberta

\section{Lisa B. Prichard}

MacEwan University

Pranidhi Baddam

University of Alberta

Daniel Graf

University of Alberta

Ordan J. Lehmann

University of Alberta

Andrew J. Waskiewicz ( $\nabla$ aw@ualberta.ca )

University of Alberta

\section{Research Article}

Keywords: Coloboma, ophthalmology, Ocular development, periocular mesenchyme, zebrafish, BMP, TGF$\beta$

Posted Date: September 22nd, 2021

DOl: https://doi.org/10.21203/rs.3.rs-876946/v1

License: (9) This work is licensed under a Creative Commons Attribution 4.0 International License. Read Full License 


\section{Abstract}

Coloboma, a congenital disorder characterized by gaps in ocular tissues, is caused when the choroid fissure fails to close during embryonic development. Several loci have been associated with coloboma, but these represent less than $40 \%$ of those that are involved with this disease. Here, we describe a novel coloboma-causing locus, BMP3. Whole exome sequencing and Sanger sequencing of patients with coloboma identified three variants in $B M P 3$, two of which are predicted to be disease causing. Consistent with this, bmp3 mutant zebrafish have aberrant fissure closure. bmp3 is expressed in the ventral head mesenchyme and regulates phosphorylated Smad3 in a population of cells adjacent to the choroid fissure. Furthermore, mutations in $b m p 3$ sensitize embryos to Smad3 inhibitor treatment resulting in open choroid fissures. Micro CT scans and Alcian blue staining of zebrafish demonstrate that mutations in bmp3 cause midface hypoplasia, suggesting that $b m p 3$ regulates cranial neural crest cells. Consistent with this, we see active Smad3 in a population of periocular neural crest cells, and bmp3 mutant zebrafish have reduced neural crest cells in the choroid fissure. Taken together, this data suggests that Bmp3 controls Smad3 phosphorylation in neural crest cells to regulate early craniofacial and ocular development.

\section{Introduction}

Vertebrate ocular development is a deeply conserved and highly coordinated developmental process. As such, even minor perturbations can lead to ocular malformations which, in many cases, are blinding. During gastrulation, retinal tissues in the eye field evaginate to form bilateral optic vesicles (Zuber et al. 2003). These vesicles migrate laterally until they contact the overlying ectoderm and induce lens formation. Subsequent coordinated invagination of the vesicles forms the bi-layered structure of the optic cup, with the lens-facing layer forming the neural retina and the lens-averted layer becoming the retinal pigmented epithelium (Schmitt and Dowling 1994; Kagiyama et al. 2005; Kwan et al. 2012). During optic cup invagination, a transient opening forms on the ventral side of the optic cup known as the optic or choroid fissure (Schmitt and Dowling 1994; Kwan et al. 2012). The choroid fissure acts as a conduit for the early embryonic ocular vasculature and optic nerve, and closure of the choroid fissure is necessary for normal ocular development (Schmitt and Dowling 1994). Failure of choroid fissure closure results in gaps in ocular tissues such as the iris and retina (Shah et al. 2012). In humans, this congenital ocular malformation is referred to as coloboma and is present in 2-19 of 100,000 live births (Williamson and FitzPatrick 2014; ALSomiry et al. 2019; Patel and Sowden 2019; Yoon et al. 2020). Coloboma and its related disorders microphthalmia (reduced ocular size) and anophthalmia (absence of one or both eyes), frequently described as MAC, account for up to $11 \%$ of cases of pediatric blindness (Yoon et al. 2020).

Although coloboma can be caused by changes to environmental factors during gestation, it is primarily caused by mutations in genes necessary for ocular development (Williamson and FitzPatrick 2014; ALSomiry et al. 2019; Patel and Sowden 2019; Yoon et al. 2020). To date, more than 40 colobomacausing loci have been identified (ALSomiry et al. 2019; Yoon et al. 2020). These genes have well characterized roles in critical developmental processes essential to choroid fissure closure, including axial 
patterning of the optic cup (proximal-distal, nasal-temporal and dorsal-ventral), cell movement and shape changes, cell adhesion, extracellular matrix remodeling, and apoptosis (ALSomiry et al. 2019; Yoon et al. 2020). Despite the substantial genetic heterogeneity, the majority of patients with coloboma do not have mutations in any known loci (Yoon et al. 2020). In addition, coloboma exhibits highly variable inheritance, with instances of coloboma pedigrees that display incomplete penetrance and reduced expressivity, suggesting that modifier loci, environmental factors, and stochastic developmental events may confound the identification of disease-causing loci. This is further complicated by marked phenotypic heterogeneity with a diverse spectrum of severity, involvement of distinct ocular structures, and frequent unilateral disease (Morrison et al. 2002; Hornby et al. 2003; Shah et al. 2012; Prokudin et al. 2014).

Of the loci that regulate choroid fissure closure, a significant proportion belong to the Transforming Growth Factor Beta (TGF-b) superfamily of signaling ligands (Williamson and FitzPatrick 2014; ALSomiry et al. 2019; Patel and Sowden 2019; Yoon et al. 2020). All TGF-b superfamily members contain three centralized disulfide bonds termed a "cysteine knot", as well as an additional disulfide bond that allows for homo- or heterodimerization of ligands (Goebel et al. 2019). This cysteine knot structure and additional disulfide bond are essential for the secretion, stability, and function of these ligands, and loss of this structure severely impairs the activity of these ligands (Goebel et al. 2019). Binding of the extensive family of TGF-b ligand dimers to a smaller cohort of tetrameric complex of Type I and Type II receptors triggers the phosphorylation of receptor-associated Sma- and Mad-related (r-Smad) proteins (Wrana et al. 1992; Bassing et al. 1994; Zhang et al. 1996). Phosphorylated r-Smad recruits co-Smad4, and this complex translocates to the nucleus, where it regulates target gene expression (Liu et al. 1996). TGF-b ligands can be divided into two groups: the Bone Morphogenetic Protein (BMP) subgroup, which typically trigger the phosphorylation of Smad1/5/8, and the Activin/Nodal/TGF-b subgroup, which predominantly induce the phosphorylation of Smad2/3 (Graff et al. 1996).

Of the two groups of TGF-b ligands, BMPs have been studied most extensively in ocular development and disease. Mutation of BMP pathway members (including ligands, antagonists, co-receptors, receptors and Smads) in patients and/or model organisms contributes to MAC, and mutations in these pathway components are also associated with related phenotypes such as orofacial clefts, craniofacial disorders, and neural tube closure defects (Sakuta et al. 2001; Hanel and Hensey 2006; Morcillo et al. 2006; AsaiCoakwell et al. 2007, 2009; Bakrania et al. 2008; French et al. 2009; Suzuki et al. 2009; Ye et al. 2010; Wyatt et al. 2010; Abouzeid et al. 2011; Reis et al. 2011; Okada et al. 2011; Zhang et al. 2013; Beleggia et al. 2015; Pfirrmann et al. 2015; Yan et al. 2020). BMP ligands are required for ocular cell survival, maintenance of stem cell pools, migration of ocular precursors, and axial patterning of the developing retina (Peters and Cepko 2002; Adler and Belecky-Adams 2002; Morcillo et al. 2006; Asai-Coakwell et al. 2007). Yet, despite intensive investigation, a clear molecular mechanism for how these ligands regulate fissure closure or a plausible explanation for complex patterns of inheritance for genes encoding these pathway components has yet to be deduced.

Early eye development is dependent not only on the intrinsic population of optic cup neurectoderm cells, but also on a population of extraocular cells that surround the developing eye cup known as the 
periocular mesenchyme (POM). These cells, which are principally derived from neural crest and cranial mesoderm, migrate over the eye cup and through the choroid fissure and contribute to many structures of the anterior segment, including the iris stroma, the cornea, and components of the intraocular drainage system (Creuzet et al. 2003; Gage et al. 2005; Langenberg et al. 2008). POM cells have also been implicated in choroid fissure closure. Notably, neural crest cells and mesodermally-derived endothelial cells are present in the fissure at times that are critical for choroid fissure closure (James et al. 2016; Bernstein et al. 2018; Gestri et al. 2018). Embryos with no appreciable neural crest contribution have open choroid fissures, suggesting that neural crest cells are necessary for closure of the choroid fissure (Gestri et al. 2018; Bryan et al. 2020). Additionally, mutations in transcription factors and signaling molecules associated with neural crest function, including BMP7, ZIC2, FOXC1, LMX1B, ALX1, and TFAP2A, have been shown to cause coloboma, further implicating this population of cells in choroid fissure closure (Morcillo et al. 2006; McMahon et al. 2009; Skarie and Link 2009; Gestri et al. 2009; Lupo et al. 2011; Dee et al. 2012; Sedykh et al. 2017).

Here, we characterize a novel coloboma causing locus that was identified by exome sequencing a threegeneration pedigree of patients with autosomal dominant coloboma and microphthalmia. Additional variants in BMP3 were identified in unrelated MAC patients using Sanger sequencing. Use of in-silico predictions and protein secretion assays provided the first evidence that variants have deleterious effects to BMP3 protein function. Zebrafish CRISPR-generated bmp3 mutants display both delayed choroid fissure closure and altered jaw development. This prompted us to test the hypothesis that Bmp3 functions in periocular cells to regulate neural crest cell behavior. We demonstrated that bmp3 mutants display marked reduced Smad phosphorylation in periocular neural crest cells. Furthermore, overall migration of periocular neural crest cells is dramatically reduced in bmp3 mutants. Our data thus provide the first evidence of a contribution of BMP3 to coloboma and highlight the importance of signaling pathways in regulating the function of periocular neural crest during ocular development.

\section{Results}

\section{Patients with coloboma and/or microphthalmia have mutations in BMP3}

To reveal the genetic cause of coloboma in archival samples, we first performed whole exome sequencing (WES) on samples from four individuals affected by autosomal dominant coloboma and one unaffected control individual from a three-generation European pedigree (Figure 1A). Identified variants were filtered using standard criteria (Beaulieu et al. 2014). Comparison to SNP databases (allele frequency $<1 \%$ ), in-silico prediction algorithms (MutationTaster, SIFT and PolyPhen), and ocular expression patterns were used to identify rare, potentially damaging variants within genes expressed during ocular morphogenesis (Figure 1A). This strategy identified 10 variants present in the affected individual's samples (II:2, III:6, III:1, and IV:4) and absent in the unaffected individual's sample (IV:5), of which the point mutations (c.1408G $>C$ ) in $B M P 3$ represented the most promising candidate pathogenic variant (Table 1). Notably, this variant exhibited Mendelian segregation in the larger pedigree, is absent from local control DNA samples, and is absent from the National Heart, Lung and Blood Institute, 1000 
Genomes, and gnomAD databases (total of 87,888 exomes and genomes) (Table 2). In addition to this $B M P 3$ variant, the variant present in CRYAA was also an attractive candidate. CRYAA encodes Crystallin alpha A, a protein that confers light-focusing properties to the lens (Brady et al. 1997). Mutations in CRYAA cause congenital cataracts with high penetrance (Litt et al. 1998; Pras et al. 2000; Mackay et al. 2003; Richter et al. 2008; Laurie et al. 2013). A small number of patients with CRYAA mutations and MAC been reported, but most patients with CRYAA do not have any features of MAC (Beby et al. 2007; Sun et al. 2017). Such patients always present with congenital cataracts, and the coloboma present in these patients is predicted to be secondarily caused by defects of the lens (Sun et al. 2017). Since none of the patients in the pedigree from Figure 1 presented with cataracts, we reasoned that the CRYAA variant present in the family from Figure 1 is likely non-pathogenic. Therefore, the variant in BMP3 still represents the most plausible variant for causing disease in this family. This mutation alters a residue (p.Ala470Pro (A470P)) that is located between cysteines that mediate the disulfide bonding necessary to form the cysteine knot structure that is essential for TGF-b ligand stability (Figure S1). This residue is also highly conserved among vertebrate species, further suggesting that it is necessary for the stability and/or function of the mature BMP3 protein (Figure S1). Collectively, this strongly indicates that changes to this residue will deleteriously impact protein function. Given that numerous TGF-b superfamily members have essential roles in ocular development and mutations in multiple TGF-b paralogs cause coloboma, we concluded that this $B M P 3$ variant represented the most plausible pathogenic variant of those identified in this family (Lo et al. 1998; Asai-Coakwell et al. 2007; Bakrania et al. 2008; Ye et al. 2010; Abouzeid et al. 2011).

Additional BMP3 variants are present in an unrelated cohort of MAC patients.

To determine whether this exceedingly rare mutation indicates that $B M P 3$ might contribute to coloboma, we searched for additional $B M P 3$ mutations by conducting Sanger sequencing of $B M P 3$ in patients unrelated to the pedigree family. DNA samples from a cohort of individuals with microphthalmia, anophthalmia and coloboma phenotypes were collected over the past 20 years through national and international collaboration. Sanger sequencing of 154 samples identified two additional point mutations in $B M P 3$ that cause single amino acid substitutions in the mature TGF-b domain of the BMP3 protein (c.1178C>T (p.Ser393Phe) and c.1349T>A (p.Phe450Tyr)) (Figure 1A-B). The Ser393Phe variant is present at exceedingly low rates in genome databases (1/76, 069 in gnomAD), whereas the Phe450Tyr variant is present in some control individuals in several databases (1/6732 in NHBLI, 1/5000 in 1KG, and 29/76, 064 in gnomAD) (Table 2). In-silico modeling using SIFT, PolyPhen2, and MutationTaster predict that the three variants are damaging to protein function, further suggesting that these are pathogenic variants (Table 3). Subsequent use of ANOLEA to bioinformatically predict protein structure indicated that each variant would also increase the free energy required to fold the BMP3 protein (Fig S2) (Melo, F., Devos, D., Depiereux, E., Feytmans 1997). Such predictions, together with the rarity of the alleles in the general population, suggested that S393F and A470P represented pathogenic variants (Table 3). The F450Y allele, since it is present in a larger portion of the general population is less likely to represent a disease-causing variant. 
To determine whether these variants altered the stability and secretion of the BMP3 ligand, the expression of V5-tagged wildtype and variant BMP3 proteins was assayed by immunoblotting. While both wildtype and variant V5-tagged BMP3 were initially expressed in transfected COS cells, differences were observed in the secretion of the BMP3 variants. There was a reduced amount of BMP3-S393F, and complete absence of BMP3-A470P, from the tissue culture media fraction when compared to wildtype BMP3 (Figure 1C). In contrast, BMP3-F450Y was still found to be secreted at levels comparable to wildtype BMP3 (Figure 1C). When quantified and compared to the level of wildtype BMP3 protein in the culture media, the intensity of the bands corresponding to the S393F and A470P variant protein in the culture media exhibited a fold change of 0.18 and 0.038 , respectively (Figure 1D). Interestingly, the band corresponding to the F450Y variant exhibited a fold change of 1.71, indicating that more of this variant was secreted into the culture media when compared to wildtype (Figure 1D). The reduction of S393F and absence of A470P from the cell culture media suggests that these variants impact the stability of mature protein or the process by which the protein is secreted from cells, whereas the presence of BMP3-F450Y in the culture media suggests that this variant does not affect the stability or secretion of BMP3.

\section{Zebrafish bmp3 mutants have choroid fissure closure defects}

To determine whether mutation of $B M P 3$ in a vertebrate model accurately recapitulated the patient phenotype, we generated zebrafish bmp3 mutants using CRISPR/Cas9 mutagenesis. The resulting allele,

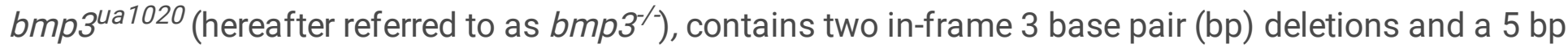
deletion (Figure S3A, Figure 2A). Each 3 bp deletion induces the loss of a single amino acid in the Bmp3 protein, while the $5 \mathrm{bp}$ deletion causes a frameshift mutation that introduces a premature stop codon immediately upstream of the sequence encoding the mature TGF-b domain (Figure S3B). This results in a truncated protein lacking the domain essential for normal BMP function (Figure 2A), and our analyses revealed no evidence that the mutant transcript undergoes nonsense mediated decay (Figure S4). Furthermore, the absence of bmp3 transcript in 2-cell stage embryos suggests that there is no compensatory maternally deposited mRNA (Figure S5)

The basement membrane surrounding the temporal and nasal retinal lobes, which must be disassembled to allow the lobes to fuse, provides an accurate readout for choroid fissure closure (Hero 1989, 1990; Tsuji et al. 2012; James et al. 2016). Therefore, the absence of the basement membrane lining the fissure is an accurate measure of fissure closure. Loss of this basement membrane is readily visualized with Laminin immunofluorescence. At $48 \mathrm{hpf}$, the stage just prior to choroid fissure closure, both wildtype and mutant choroid fissures were open (Figure 2B-C).

In contrast, at $65 \mathrm{hpf} 18 \%(23 / 130)$ of $b m p 3^{-/}$mutant embryos retained the basement membrane surrounding the fissure compared to $0 \%(0 / 38)$ of wildtype controls demonstrating that $b m p 3$ contributes to normal choroid fissure closure (Figure 2D-F). Statistical analysis (two-sided Fisher's exact test) confirmed that open choroid fissures occur more frequently in $b m p 3^{-/-}$mutants when compared to 
wildtype animals at $65 \mathrm{hpf}(\mathrm{p}=0.0025$, Fisher's exact test). These data also provide evidence that the bmp $3^{- \text {c }}$ choroid fissure phenotype is partially penetrant, as is observed in some coloboma pedigrees (Figure S6).

bmp3 is expressed in the ventral head mesenchyme surrounding the eye

To explore the mechanisms by which bmp3 facilitates choroid fissure closure, the spatial expression of bmp3 mRNA was assessed. The earliest localized cranial expression of bmp3 begins at approximately 22 $\mathrm{hpf}$, with dorsal flat mounts demonstrating bmp3 is expressed in the vicinity of the forebrain and the eye (Figure $3 \mathrm{~A}$ ). Cranial coronal sections reveal that $b m p 3$ is expressed in a single-cell thick layer in the head mesenchyme immediately ventral to the eye (Figure 3B). Transverse sections of the head further confirm bmp3 expression in the head mesenchyme between the brain and retina and not within the neuroepithelial cells of the retina or brain (Figure $3 \mathrm{C}$ and $\mathrm{D}$ ). In conclusion, bmp3 is expressed in the ventral head mesenchyme adjacent to the choroid fissure, a cell population that is composed of POM cells.

\section{Bmp3 does not regulate dorsal-ventral patterning of the early eye}

During ocular morphogenesis, the eye must be patterned along the dorsal-ventral axis (Peters and Cepko 2002). This is achieved through opposing gradients of dorsally-restricted targets of BMP signaling and ventrally-restricted targets of Sonic Hedgehog signaling (Peters and Cepko 2002). To determine whether bmp3 facilitates dorsal-ventral axis patterning of the optic cup, the spatial expression of genes that are restricted to either the dorsal (tbx5a, aldh1a2) or the ventral (vax2, aldh1a3) regions of the eye were examined in wildtype and $b m p 3^{-/-}$mutants using in-situ hybridization (Peters and Cepko 2002). In wildtype embryos, the expression of dorsal markers is restricted to the dorsal half of the optic cup, and ventral markers are restricted to the ventral optic cup, as expected $(n=25)$ (Figure 4A, C, E, G). Identical expression patterns are observed in $b m p 3^{/-}$mutants $(n=25)$ (Figure 4B, D, F, H), suggesting that $b m p 3$ does not regulate axial patterning of the retina.

\section{Bmp3 does not regulate proliferation or apoptosis in the early eye}

In addition to dorsal-ventral patterning of the optic cup, aberrancies in optic cup cell proliferation and cell death have also previously been shown to contribute to the appearance of a coloboma phenotype in several animal models (Kim et al. 2007; Viringipurampeer et al. 2012; Lee et al. 2013; Van Nostrand et al. 2014; Noh et al. 2016; Sghari and Gunhaga 2018). Therefore, it is plausible that $b m p 3$ regulates proliferation or apoptosis of neuroectodermal optic cup cells, and the loss of bmp3 results in aberrant proliferation or apoptosis, thereby leading to choroid fissure closure defects. To determine whether bmp3 regulates cell proliferation or apoptosis, immunofluorescence was conducted on 28 hpf wildtype and $b m p 3^{--}$embryos using primary antibodies against phosphorylated Histone $\mathrm{H} 3$ (to assay for proliferation) or activated Caspase 3 (to assay for apoptosis). We do not observe an obvious change in proliferation of the optic cup in $b m p 3^{-/}$mutants $(\mathrm{n}=20)$ when compared to wildtype controls $(\mathrm{n}=20)$ 
(Figure $4 \mathrm{I}, \mathrm{J}$ ), indicating that $b m p 3$ does not regulate proliferation of the optic cup. Likewise, there is no obvious change in apoptosis of the optic cup between $b m p 3^{-/-}$mutants $(\mathrm{n}=19)$ when compared to wildtype controls $(\mathrm{n}=19)$ (Figure $4 \mathrm{~K}, \mathrm{~L})$, indicating that bmp3 does not regulate apoptosis of cells in the optic cup. Taken together, these results indicate that bmp3 does not regulate proliferation or apoptosis of the optic cup cells.

\section{Bmp3 facilitates choroid fissure closure via Smad3 phosphorylation}

The majority of BMP ligands signal via the phosphorylation of Smad1/5/8 rather than Smad2/3 phosphorylation, which is typical of other TGF-b family members such as Activin and Nodal (Miyazono et al. 2000). Interestingly, BMP3 has been shown to activate Smad2/3 rather than Smad1/5/8, suggesting it functions in a noncanonical manner compared to other BMP ligands (Bahamonde and Lyons 2001; Daluiski et al. 2001; Stewart et al. 2010). To determine the level of Smad3 phosphorylation adjacent to the choroid fissure, immunofluorescence using an anti-phosphorylated Smad3 (pSmad3) primary antibody was first assessed in wildtype embryos. Confocal imaging revealed active phosphorylated Smad3 restricted to cells in the proximal portion of the nasal lobe, adjacent to the choroid fissure (Figure $5 A)$. In contrast, $z$-stacks from the medial to distal regions of the eye demonstrated little detectable pSmad3 (Figure 5B, C). In conclusion, this indicates that pSmad3 is present in cells that are positioned optimally to facilitate fissure closure.

We next assessed the presence of pSmad3 in wildtype and $b m p 3^{\%}$ embryos to determine whether Bmp3 facilitates choroid fissure closure via Smad3 phosphorylation. While the distinct population of pSmad3 positive cells adjacent to the choroid fissure are present in both wildtype and $b m p 3^{-/-}$embryos (Figure 5D, E), there was a subtle, yet statistically significant, reduction in the number of cells containing phosphorylated Smad3 in the ventro-proximal retina of $\mathrm{bmp3}^{-/}$mutants (18.3 \pm 1.09 cells) when compared to wildtype siblings ( $22.2 \pm 1.12$ cells) (two-sample t-test, $t_{38}=2.49, p=0.0172$ ) (Figure $5 F$ ). Since compensation by other TGF-b ligands represents one explanation for the subtlety of the pSmad3 phenotype, we treated $b m p 3^{--}$mutants with the pharmacological agent Specific Inhibitor of Smad3 (SIS3) and measured choroid fissure closure. SIS3 was titrated to yield an incompletely penetrant phenotype, and the suboptimal dose selected (12 mM SIS3) yielded open choroid fissures in $15-20 \%$ of wildtype embryos. SIS3 treatment from 24-30 hpf resulted in a significantly higher proportion of open choroid fissures in bmp $3^{-/}$mutants compared to wildtype embryos (bmp $3^{-2}$ 23/31 (74\%), WT 5/28 (18\%), two proportion $z$-test, $\mathrm{z}=-4.33, \mathrm{p}<0.00001$, Figure $5 \mathrm{G}-\mathrm{I})$. In summary, these data show that $b m p 3$ mutation synergizes with pharmacological inhibition of Smad3 to impede choroid fissure closure.

\section{Smad3 phosphorylation is active in neural crest cells in the choroid fissure}

POM cells are derived from neural crest cells and cranial mesoderm and have previously been shown to be necessary for choroid fissure closure (James et al. 2016; Gestri et al. 2018; Bryan et al. 2020). In a study of canine brachycephaly, the loss of $B M P 3 / b m p 3$ during development results in craniofacial phenotypes including the loss or hypoplasia of early craniofacial cartilage in certain dog breeds and 
morphant zebrafish (Schoenebeck et al. 2012). This is consistent with our own observations of adult bmp $3^{-/-}$mutants. Midfacial hypoplasia is present in 18-month-old adult $b m p 3^{\%-}$ zebrafish and is visible under a dissecting microscope (Fig. 6A, B). This is further confirmed by Micro-Computerized Tomography (Micro-CT) scans of adult wildtype and $b m p 3^{--}$mutants; $b m p 3^{-/-}$mutants $(\mathrm{n}=5)$ have visually altered craniofacial anatomy when compared to wildtype siblings $(n=5)$ (Figure 6A-D). When quantified, bmp $3^{\%}$ mutants display significantly shortened average pre-maxilla $(1.20 \pm 0.167 \mathrm{~mm})$, maxilla $(1.58 \pm 0.0676 \mathrm{~mm})$, and mandible $(2.23 \pm 0.112 \mathrm{~mm})$ length when compared to wildtype siblings $\left(1.58 \pm 0.250 \mathrm{~mm}, 1.88 \pm 0.0823\right.$, and $2.68 \pm 0.146 \mathrm{~mm}$, respectively) (two sample t-test, $\mathrm{t}_{8}=2.85,6.22$, and 5.50, p =0.000255, 0.0214, and 0.000573) (Figure 6G-I). Additionally, analysis of $7 \mathrm{dpf}$ larval zebrafish jaws stained with Alcian blue revealed that $40 \%(\mathrm{n}=8 / 20)$ of $b m p 3^{-/}$embryos display jaws that are hypoplastic when compared to wildtype controls $(n=0 / 20)$ (Figure $6 \mathrm{~J}, \mathrm{~K})$. The morphology of the chondrocytes that make up Meckel's cartilage are also severely compromised in $\mathrm{bmp} 3^{-/}$mutants; the chondrocytes of Meckel's cartilage in affected $b m p 3^{-/}$mutants appear rounder, undifferentiated, and less organized when compared to Meckel's cartilage from wildtype animals, where the chondrocytes are elongated, stacked, and columnar (Figure $6 \mathrm{~J}^{\prime}, \mathrm{K}^{\prime}$ ). This evidence implicates $b m p 3$ as a regulator of craniofacial development. Since all craniofacial cartilage and bone is derived from neural crest cells, and since there is no change in patterning, proliferation, apoptosis and size of the optic cup in $b m p 3^{-/-}$mutants, we hypothesize that $b m p 3$ regulates neural crest cell-mediated choroid fissure closure.

To determine whether pSmad3 is present in cells in the vicinity of periocular neural crest cells, we performed pSmad3 immunofluorescence on $\operatorname{Tg}($ sox 10:EGFP) embryos, a strain that specifically labels migratory neural crest cells. It has previously been shown that periocular neural crest cells are readily apparent in the choroid fissure at 28-30 hpf (James et al. 2016; Bernstein et al. 2018; Gestri et al. 2018). Therefore, to assess the state of phosphorylated Smad3 in the context of neural crest cells, pSmad3 immunofluorescence was conducted on wildtype $\operatorname{Tg}($ sox 10 :EGFP) embryos at 24,28 , and $32 \mathrm{hpf}$. We observe neural crest cells in close proximity to the choroid fissure and in the choroid fissure itself at 24 , 28, and $32 \mathrm{hpf}$ (Figure 7A, D, and G). At $24 \mathrm{hpf}$, neural crest cells have initiated infiltration of the choroid fissure (Figure 7A). At this time, there is also a small amount of retinal pSmad3 in the proximal retina, suggesting that $24 \mathrm{hpf}$ is one of the earliest stages when TGF-b signaling is active in the choroid fissure (Figure 7B). Moreover, the appearance of this early population of neural crest cells coincides with the first appearance of pSmad3 in the retina (Figure 7C). At $28 \mathrm{hpf}$, the choroid fissure and ventral eye have become highly infiltrated with neural crest cells, and within the choroid fissure neural crest cells appear to completely wrap around both lobes of the choroid fissure, namely the nasal lobe (Figure 7D), and pSmad3 is readily detectable in the ventro-nasal retina and ventral POM (Fig 7E, F). At 32 hpf, neural crest cells are still present in the choroid fissure in numbers comparable to that of $28 \mathrm{hpf}$ (Figure 7G). A fraction of these cells have migrated dorsally behind the lens, where they form pericytes that line the hyaloid vasculature that forms here (Figure 7G) (Trost et al. 2013). At this stage, ventro-nasal retinal pSmad3 has faded; the only detectable pSmad3 in the eye corresponds to the proximal lens tissue (Figure $7 \mathrm{H}$ ). There is no longer a clear domain of neural crest cells that colocalize with the pSmad3 signal 
(Figure 7J). Taken together, this data suggests that neural crest cells may be regulated by TGF-b ligands in the ventro-nasal retina at $28 \mathrm{hpf}$.

\section{Bmp3 regulates neural crest cell numbers in the choroid fissure}

Given that Smad3 phosphorylation is active in neural crest cells and that Bmp3 appears to regulate fissure closure vis Smad3 phosphorylation, we hypothesized that Bmp3 is a regulator of periocular neural crest function. To test this, we crossed our $\mathrm{bmp3}^{-/}$mutant line to our $\mathrm{Tg}$ (sox10:EGFP) transgenic line of zebrafish, which fluorescently label neural crest cells. In wildtype embryos, we observe many neural crest cells lining the fissure, particularly around the nasal lobe at $28 \mathrm{hpf}$ (Figure $7 \mathrm{~J})(\mathrm{n}=18$ eyes). In contrast, there are visibly fewer neural crest cells in the ventral eye of $b m p 3^{-/}$mutant embryos (Figure 7K) $(n=18$ eyes). When quantified, the average number of neural crest cells in the choroid fissure is significantly reduced in $b m p 3^{-/}$mutants ( $6.56 \pm 1.04$ cells) when compared to wildtype animals (10.0 \pm 1.00 cells) (student's t-test, $t_{34}=4.76, p<0.0001$, Figure $7 \mathrm{~L}$ ). This suggests that $B m p 3$ regulates the number of neural crest cells in the choroid fissure at $28 \mathrm{hpf}$, a timepoint that is critical for choroid fissure morphogenesis and closure.

\section{Discussion}

Despite being a significant cause of pediatric blindness, the genetic origins of coloboma are still incompletely understood. Here, we identify and characterize the role of BMP3 in ocular morphogenesis and coloboma for the first time. Using both human patient data and zebrafish experimental evidence, we show that $B M P 3$ regulates choroid fissure closure through non-canonical Smad3 phosphorylation. We also provide evidence for $B M P 3$ regulating choroid fissure closure via the periocular mesenchyme, a process that remains poorly understood.

Studies performed using human patient data and model organisms have identified a number of loci that are involved in coloboma, collectively referred to as "the coloboma gene network" (Williamson and FitzPatrick 2014). Notably, genes that are overrepresented in this network encode components and downstream targets of developmental signaling pathways. This includes the TGF-b/BMP, sonic hedgehog, retinoic acid, fibroblast growth factor, and Wnt pathways (ALSomiry et al. 2019; Patel and Sowden 2019). This overrepresentation highlights the importance of developmental signaling pathways in ocular development, choroid fissure closure, and organ morphogenesis in general. This, in part, led us to choose $B M P 3$ as a candidate for investigation; BMPs have well characterized roles in the patterning of multiple organs, including the eye (Peters and Cepko 2002). Although some other candidates identified by WES are expressed in the developing eye, we were able to systematically eliminate them as candidates, as mutations in these genes are not significant contributors to MAC. Moreover, BMP3 remained the most likely candidate due to its previously established role as a developmental signaling molecule (Bahamonde and Lyons 2001; Daluiski et al. 2001). In addition to the BMP3 variant identified by our WES experiments (A470P), the two additional BMP3 variants (S393F and F450Y) identified from Sanger sequencing 154 unrelated MAC patients strongly suggests that $B M P 3$ is a locus involved in the etiology 
of coloboma. These variants are extremely rare, suggesting that they are not benign variants found in the general population; rather, it is likely that these variants contribute to the etiology of MAC in these patients. This is supported by both predictive algorithms of protein folding and cell secretion assays, which show that at least two of the variants, A470 and S393, result in the absence of these variant proteins from the media of COS-7 cells. Both A470 and S393 are located near cysteine residues that participate in the disulfide bonds that are critical for the stability of mature BMP3 dimers. Therefore, the presence of amino acid substitutions at either of these residues may result in aberrant cysteine knot formation, resulting in lowered extracellular stability of BMP3 and thus its absence from the cell culture media (Goebel et al. 2019). Interestingly, there is another variant at the A470 residue listed in gnomAD (p.Ala470Thr) that is present in control individuals (7/75 435 individuals). Although this may suggest that change to the A470 residue do not cause changes to BMP3 activity, the A470T amino acid change is, relative to A470P, conservative; threonine is small and uncharged, making it unlikely that it dramatically affects BMP3 protein structure and function. Proline, on the other hand, forms a pyrrolidine loop with the nitrogen from the amino acid backbone. This pyrrolidine loop has previously been observed to dramatically disrupt the secondary structure of proteins, therefore making it exceptionally likely that this amino acid disrupts BMP3 protein structure (Levitt 1978). Taken together, this evidence strongly suggests that the patient variants identified through WES and Sanger sequencing have deleterious consequences to protein activity, further implicating $B M P 3$ as a novel coloboma-causing locus.

For the third variant detected, F450Y, we do not observe any secretion aberrancies. This residue is located in close proximity to the receptor-binding domain of the BMP3 protein (Allendorph et al. 2007). The receptor binding domain of BMP3 is extremely sensitive to amino acid composition, and single amino acid substitutions have been shown to reduce its affinity for its receptor (Allendorph et al. 2007). Alternatively, this residue may not be pathogenic; this is supported by the presence of this variant in control genome databases (Table 2). Therefore, more investigation is needed to confirm whether BMP3F450Y is a pathogenic variant.

We show that zebrafish $\mathrm{bmp}^{-/}$mutants have an incompletely penetrant coloboma phenotype. Additionally, we show that this phenotype is a delay in fissure closure, as all $\mathrm{bmp} \mathrm{3}^{- \text {- }}$ mutants assayed have closed fissures at $72 \mathrm{hpf}$. The nature of the choroid fissure closure defects observed in these mutants may give some indication as to $b m p 3$ 's role in fissure closure. It has been proposed that coloboma can be broadly separated into two classes: morphogenetic coloboma, which arises from defects the morphogenesis of the eye cup, and fusion defective coloboma, which arises from defects in degradation of the basal lamina or adhesion of the retinal cells that make up the choroid fissure (James et al. 2016; Eckert et al. 2020). Eyes from $b m p 3^{-/}$mutants are not smaller in size than those from wildtype embryos, and the fissure lobes are fully apposed without any gross morphological defects, suggesting that bmp3 is not a regulator of early ocular morphogenetic events such as optic vesicle evagination or choroid fissure formation, as perturbations to these process usually cause overt morphological defects such as microphthalmia and severe, very obvious coloboma (Patel and Sowden 2019). Due to the subtlety of the choroid fissure closure defects in bmp3 mutants, it is more likely that 
bmp3 facilitates processes that occur later in choroid fissure closure, such as basement membrane breakdown or intercalation of the retinal cells that make up the lobes of the choroid fissure. Indeed, the phenotypes of bmp3 mutants resemble other zebrafish models of defective basement membrane degradation and cellular intercalation, further suggesting that $b m p 3$ plays a role in these processes (James et al. 2016; Hardy et al. 2019).

Previous studies of bmp3 function have shown that it non-canonically activates the phosphorylation of Smad2/3 rather than Smad1/5/8 (Bahamonde and Lyons 2001; Daluiski et al. 2001; Stewart et al. 2010). We show that $b m p 3$ is likely facilitating choroid fissure closure through this mechanism, as indicated by

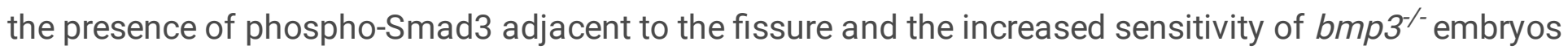
to SIS3. Recent work on zebrafish has shown that there are other TGF-b ligands expressed in the fissure, and that TGF-b signaling is active in the fissure at the same time we detect phospho-Smad3 in the retina (Knickmeyer et al. 2020). Therefore, bmp3 may act in concert with other TGF-b ligands to facilitate choroid fissure closure (Knickmeyer et al. 2020). The mechanism behind this process is still not clear; others have proposed that TGF-b signaling via Smad2/3 phosphorylation in the fissure promotes the expression of BMP antagonists which leads to the inhibition of dorsal BMP signaling (Knickmeyer et al. 2020). This thereby creates a restricted domain of BMP expression in the dorsal eye and conferring dorsal identity (Knickmeyer et al. 2020). Indeed, BMP3 has been shown to inhibit both canonical BMP signaling and Activin signaling (Gamer et al. 2005; Knickmeyer et al. 2020). This mechanism has also been well documented in other developmental processes including axis patterning and gastrulation (Little and Mullins 2006). However, this is somewhat at odds with our results; we do not observe any changes to the expression of dorsal markers, especially $t b x 5 a$, which is a direct transcriptional output of BMP signaling in the dorsal eye, suggesting that $b m p 3$ doesn't antagonize dorsal BMP signaling. Therefore, more research is needed to determine the precise pathway through which Bmp3 inhibits canonical BMP signaling in eye development.

Interestingly, bmp3 is not expressed in the dorsal eye like other ocular BMP ligands, such as gdf6a (French et al. 2009; Gosse and Baier 2009). Rather, it is expressed in the ventral head mesenchyme adjacent to the fissure. The head mesenchyme is populated by both cranial neural crest cells and paraxial and splanchnic mesoderm (Creuzet et al. 2003; Gage et al. 2005). Accordingly, there has been evidence that $b m p 3$ functions in both the mesoderm and the cranial neural crest; $b m p 3$ expression has been detected in the tail paraxial mesoderm of zebrafish and has also been shown to be a regulator of neural crest-derived craniofacial structures (Mueller et al. 2010; Schoenebeck et al. 2012). Here, we provide evidence that $b m p 3$ is a regulator of cranial neural crest cells rather than cranial mesoderm, as indicated by microCT scans of adult fish and Alcian blue staining of larval jaw cartilage. Although this is consistent with other reports of bmp3 function, we find that the larval mutant phenotypes are far less severe and have lower penetrance than previously reported (Schoenebeck et al. 2012). However, these previous studies were performed using morpholino oligonucleotides, which have been shown to produce more severe phenotypes when compared to mutants (Eve et al. 2017). Regardless, we provide further evidence that $b m p 3$ regulates neural crest-derived structures and is novel regulator of craniofacial development, 
particularly structures that contribute to the jaw. This is somewhat unsurprising, as craniofacial abnormalities and coloboma commonly occur together in syndromes including CHARGE syndrome and BOFS syndrome (Siebert et al. 1985; Gestri et al. 2009). These studies, together with our own studies of $B M P 3$ 's involvement in coloboma, suggest that there is likely a shared neural crest-derived etiology of coloboma and craniofacial abnormalities in many contexts (Gestri et al. 2009; Bajpai et al. 2010). Moreover, it wouldn't be entirely surprising to find craniofacial abnormalities in patients with damaging mutations in $B M P 3$, given that we see craniofacial abnormalities in our zebrafish model. In our studies of cartilage development in $b m \mathrm{p}^{-/}$mutants, we observe jaw chondrocytes that are less organized and undifferentiated when compared to wildtype controls, suggesting that bmp3 doesn't affect the migration of neural crest cells to form the cartilage, but rather regulates the differentiation of cranial neural crest cells into chondrocytes. Moreover, only anterior structures derived from pharyngeal arch 1 and 2 (such as Meckel's cartilage, the palatoquadrate, and the ceratohyal) are affected, whereas structures derived from the more posterior pharyngeal arches 3-7 (such as the ceratobranchials) remain largely unaffected, suggesting that $b m p 3$ 's role on craniofacial development is restricted to the neural crest cells of the first pharyngeal arch; indeed, other studies of bmp3's role on craniofacial development found its expression to be highest in the anterior pharyngeal arches and lower or absent in the more posterior arches (Schoenebeck et al. 2012). This suggests that bmp3 may be regulating the differentiation of arch 1 and 2 neural crest cells, and the absence of bmp3 in the first arch results in undifferentiated chondrocytes and, thus, the craniofacial defects we observe in $b m p 3^{-/}$mutants. Alternatively, $b m p 3$ could be regulating cranial neural crest cell division, survival, or death; a lack of bmp3 results in an incorrect number of chondrocyte precursors, which, ultimately, results in disorganization of the cartilage seen in bmp3 mutants. Consistent with this, there appears to be more chondrocytes present in the jaws of bmp $3^{-/}$mutants. Once the cranial neural crest cells condense into cartilage, convergence extension elongates the chondrocytes and facilitates chondrocyte stacking (Kimmel et al. 1998). Other studies have shown that disrupting genes necessary for convergence extension results in jaw phenotypes similar to bmp $3^{--}$mutants, suggesting that $b m p 3$ could have a role in regulating the number of cells necessary for convergence extension to occur properly (Rochard et al. 2016). Further research is needed to establish the precise role of $b m p 3$ in craniofacial development and cranial neural crest function.

In addition to regulating the neural crest-derived craniofacial skeleton, we also show that $b m p 3$ regulates periocular neural crest cells during choroid fissure closure. There have been several studies that have shown that these populations of cells are necessary for choroid fissure closure, further solidifying the role of these cells as necessary mediators of choroid fissure closure (James et al. 2016; Gestri et al. 2018). This is also supported by our studies, where ventral periocular neural crest cells colocalize with pSmad3 signal, suggesting that neural crest cells are responsive to TGF-b signaling. This is in agreement with other studies of periocular neural crest cells and TGF-b signals, which show that neural crest-specific deletion of Tgfbr2 results in compound ocular defects in mice (Ittner et al. 2005). Therefore, this represents a plausible mechanism for TGF-b signaling in the eye, where cranial neural crest cells are responsive to Bmp3 and potentially other TGF-b ligands. This also fits with our model of Bmp3 facilitating later stages of choroid fissure closure; although some reports of neural crest-mediated 
choroid fissure closure have shown that periocular neural crest cells are necessary for early eye vesicle morphogenesis, others have proposed that neural crest cells are necessary for later stages of choroid fissure (James et al. 2016; Gestri et al. 2018; Hardy et al. 2019; Bryan et al. 2020). We also observe a reduction in the number of neural crest cells in the choroid fissures of $b \mathrm{mp}^{-/-}$mutants at $28 \mathrm{hpf}$, suggesting that Bmp3 regulates the number of neural crest cells in the choroid fissure. However, it is unclear whether Bmp3 regulates neural crest number, guidance, or differentiation. Previous reports and our own studies of Bmp3 function in craniofacial development have shown that the neural crest-derived craniofacial cartilage is severely reduced in bmp3 morphants, suggesting that $b m p 3$ plays a role in neural crest differentiation (Schoenebeck et al. 2012). In contrast, other reports of periocular neural crest migration suggest that TGF-b signals emanating from the lens regulate periocular neural crest migration into the anterior segment (Takamiya et al. 2020). Therefore, it is plausible that ocular TGF-b signals are also necessary for facilitating migration of periocular neural crest cells into the choroid fissure.

In summary, we present the discovery of a novel locus involved with choroid fissure closure, BMP3. We have shown that BMP3 likely facilitates fissure closure through Smad3 phosphorylation, and we also provide evidence that BMP3 may regulate periocular neural crest cells during the process of fissure closure. Although the present study has advanced our understanding of choroid fissure closure biology and, thus, coloboma, further work will need to be done to identify additional loci that are involved in this process. Additionally, more investigation is warranted to identify and characterize loci that are expressed by the periocular mesenchyme and their role in choroid fissure closure as well as paracrine factors that regulate the periocular mesenchyme which will, in turn, inform our understanding of vertebrate ocular morphogenesis.

\section{Materials And Methods}

\section{Ethics Statement}

Whole exome and Sanger sequencing of human patient DNA

Whole exome sequencing (WES) was performed on genomic DNA from each proband as

part of FORGE Canada Consortium at the McGill University and Genome Quebec Innovation Centre. Exome target enrichment was performed using the Agilent SureSelect 50Mb (V3) All Exon Kit and sequencing was performed on the Illumina HiSeq 2000, multiplexing three samples per lane. The mean coverage of coding sequence regions, after accounting for duplicate reads was greater than 70x. WES data was analyzed by performing alignment with BWA, duplicate read removal with Picard, local indel realignment with GATK, variant calling with SAMtools, and annotation with Annovar and custom scripts.

For Sanger sequencing of the MAC patient cohort unrelated to individuals in the pedigree from Figure 1, intronic primers were used to amplify BMP3 in 154 patients with MAC. The amplicons were then purified 
and sent for Sanger sequencing at the at the Genome Quebec Innovation Centre.

\section{Zebrafish husbandry}

Adult zebrafish were kept on a 10-hour dark/14-hour light schedule. Embryos were raised at $25.5^{\circ} \mathrm{C}$, $28.5^{\circ} \mathrm{C}$ or $33^{\circ} \mathrm{C}$ in embryo media and staged according to standardized developmental hallmarks (Kimmel et al. 1995). Embryos that were grown past $24 \mathrm{hpf}$ were treated with 0.004\% 1-phenyl 2-thiourea (PTU, Sigma Aldrich P7629) prior to $22 \mathrm{hpf}$ to block pigment formation. Anesthesia of larval and adult zebrafish was performed with a $4 \%$ dilution of $0.4 \%$ tricaine methanesulfonate (TMS) stock solution (Sigma Aldrich E10521). The AB wildtype strain, the $b m p 3^{\%-}$ mutant line, and the $T g$ (sox10:EGFP) transgenic line were used. The $b m p 3^{-/-}$line contains two in-frame 3 basepair (bp) deletions (NM_001077765.1:c.543_545del and NM_001077765.1:c.840_842del) and a 5bp deletion (NM_001077765.1:c.886_890del). Both of the 3bp deletions cause the deletion of a single glutamine residue (NP_001071233.1:p.GIn182del and NP_001071233.1:p.Gln281del). The 5bp results in a frameshift and premature stop codon upstream of the mature TGF-b signaling domain (NP_001071233.1:p.Thr296GlyfsTer2).

\section{Atomic Non-Local Environment Assessment (ANOLEA)}

Using the previously solved crystal structure for BMP3, the effect of patient amino acid substitutions on protein function were modeled in silico using Swiss-pbd Veiwer and analyzed using the ANOLEA server (http://melolab.org/anolea/) (Melo, F., Devos, D., Depiereux, E., Feytmans 1997; Allendorph et al. 2007). Using the ANOLEA data, the difference in energy requirement needed to fold the BMP3 protein with each amino acid substitution was plotted in Microsoft Excel.

\section{Site-directed mutagenesis}

The open reading frame for wildtype human $B M P 3$ was obtained from ASU BioDesign Institute in pDNRDual and was moved into pcDNA3.2/V5. Oligonucleotide primers for site-directed mutagenesis were designed to generate constructs carrying c.1178C $>T$ (p.S393F), c.1349T>A (p.F450Y), and c.1408G >C (p.A470P) variants individually. The PCR reaction for site-directed mutagenesis contained: $500 \mathrm{ng}$ of template plasmid DNA, $17 \mu \mathrm{l}$ of master mix (100 $\mu$ l0x PFU buffer, $792 \mu$ water, $2 \mu \mathrm{l}$ each of $100 \mathrm{mM}$ dNTPs), $1 \mu \mathrm{l}(10 \mathrm{mM}$ ) NAD, $0.5 \mu \mathrm{l}(200 \mathrm{ng})$ each primer, $0.5 \mu \mathrm{l}$ DMSO, $0.3 \mu \mathrm{l}$ Taq DNA Ligase (NEB, M0208S), $1 \mu \mathrm{l} \mathrm{PfuUltra} \mathrm{DNA} \mathrm{Polymerase} \mathrm{(Agilent,} \mathrm{600670),} \mathrm{and} 1.5 \mu \mathrm{l}$ water. The PCR cycle conditions were denaturation at $95^{\circ} \mathrm{C}$ for 2 mins, then 30 cycles of $95^{\circ} \mathrm{C}$ for $1 \mathrm{~min}, 55^{\circ} \mathrm{C}$ for $1 \mathrm{~min}$, and $65^{\circ} \mathrm{C}$ for 10 mins. PCR products were then digested with $1 \mu \mathrm{l}$ of Dpnl (NEB, R0176S) for 30 mins at $37^{\circ} \mathrm{C}$ to digest the original plasmid. Constructs were verified by Sanger sequencing to ensure the mutation was introduced correctly. DNA for the four constructs was isolated by maxi prep (Qiagen, 12165) and Sanger sequenced to confirm the mutation sites.

Tissue Culture and Immunoblotting 
COS-7 cells were plated to 6-well dishes and transfected at $80 \%$ confluency. $3 \mathrm{mg}$ of DNA was transfected per well using Lipofectamine 2000 (ThermoFisher Scientific, 12566014) according to the manufacturer's instructions. Transfected cells were grown in low serum $(0.1 \%)$ conditions for 48 hours and then harvested. Media was removed and saved for secreted protein analysis. Cell monolayers were rinsed once in phosphate buffered saline (PBS), and $500 \mathrm{ml}$ of cold lysis buffer was added. Lysis buffer components were $50 \mathrm{mM}$ Tris, $150 \mathrm{mM} \mathrm{NaCl}, 1 \mathrm{mM}$ EDTA, $1.5 \%$ SDS, and one complete mini protease inhibitor tablet (Millipore/Sigma 11836170001), ph7.5. Cells sat on ice for 10 minutes and were then scraped and collected in Eppendorf tubes. Samples were boiled, transferred to QiaShredder tubes (Qiagen, 79656), centrifuged, and the flow-through was collected in new tubes. Proteins from media were concentrated by acetone precipitation (1:1.5 media to acetone volume) and air dried. A minimal volume of lysis buffer was added to resuspend the pellets. Protein concentration was determined using the Bradford colorimetric protein assay (BioRad, 5000201). Cell lysates and concentrated media lysates were run on 4$12 \%$ Bis-Tris gradient gels (Invitrogen NP0336) for 1 hour at 200 volts. Resolved proteins were transferred to polyvinylidene difluoride (PVDF) membranes (Millipore IPVH00010) at 22 volts for 1 hour on a semi-dry blotter (Amersham Biosciences). Blots were blocked in 5\% milk in Triton buffered saline with Tween 20 (TBST) for 2 hours and primary antibody was added for overnight incubation at $4^{\circ} \mathrm{C}$. Mouse monoclonal anti-V5 primary antibody (ThermoFisher, MA5-15253) was used at 1:5000 dilution in 5\% milk and mouse monoclonal anti-tubulin primary antibody (Sigma, T9026) was used at 1:10,000. After washing, sheep anti-mouse IgG HRP-conjugated secondary (Amersham, NA931) was added at 1:5000 for 1 hour at room temperature. Membranes were washed in SuperSignal West Pico PLUS ECL reagent (ThermoFisher, 34577) for 3 minutes and detection was performed using the ChemiDoc MP Imaging System (BioRad).

\section{CRISPR/Cas9 Mutagenesis}

Short guide RNA (sgRNA) design and synthesis were performed as previously described (Gagnon et al. 2014). The sequences for the oligonucleotides used to generate the templates for sgRNA synthesis are: 5'-GGGACTTCATCTCATGGCAGTGG-3', 5'-GGGAGCTCATTGTTCTGCAGTGG-3' and 5'GGCTGGCCTCATCCCATGTAGGG-3'. Cas9 protein (PNA Bio, CP01) was reconstituted in sterile water to a concentration of $50 \mathrm{mg} / \mathrm{mL}$ and $2 \mathrm{~mL}$ was mixed with $1 \mathrm{~mL}$ of each sgRNA. One- or Two-cell stage embryos were injected with $1 \mathrm{~nL}$ of Cas9/sgRNA mixture and allowed to develop to sexual maturity. Carriers for frameshift mutations were identified by high resolution melt (HRM) analysis using the Type-It HRM PCR kit (Qiagen, 206544) on a Rotor Gene Q qPCR machine (Qiagen, 9001560) and Sanger sequencing.

\section{Zebrafish genotyping}

$b m p 3^{-/-}$mutants were genotyped using polymerase chain reaction (PCR) followed by gel electrophoresis. Genomic DNA was extracted from fin clips in $20 \mathrm{~mL}$ of $50 \mathrm{mM} \mathrm{NaOH}$ at $95^{\circ} \mathrm{C}$ for 15 minutes and subsequently neutralized using $2 \mathrm{~mL}$ Tris- $\mathrm{HCl}, \mathrm{pH}$ 8.0. Samples were diluted $1 / 2$ in sterile water and used as template for PCR using the following primers: Forward: 5'-CTTCATATGCTGGAATCGCATAAC-3', Reverse: 5'-TTGCTCTCCATCGGATCATAAG-3'. PCR was performed using the following conditions: 
Denaturation at $94^{\circ} \mathrm{C}$ for 2 minutes, 40 cycles of $94^{\circ} \mathrm{C}$ for 15 seconds, $58^{\circ} \mathrm{C}$ for 15 seconds, and $72^{\circ} \mathrm{C}$ for 12 seconds, followed by a final extension cycle at $72^{\circ} \mathrm{C}$ for 3 minutes. PCR products were then run on a $3 \%$ agarose gel to resolve the wildtype and mutant amplicons (167bp and 156bp, respectively).

\section{In-situ hybridization}

Antisense riboprobes labeled with digoxygenin (DIG) were synthesized from purified, linearized expression plasmid containing a gene-specific insert or from a gene-specific PCR product with an integrated T7 RNA polymerase site. Probe synthesis was performed as previously described (Thisse and Thisse 2008).

Embryos were fixed overnight at $4^{\circ} \mathrm{C}$ in $4 \%$ paraformaldehyde (PFA) and subsequently permeabilized for 5 minutes at room temperature (RT) using $10 \mathrm{mg} / \mathrm{ml}$ Proteinase $\mathrm{K}$ in PBST. In-situ hybridization was performed as previously described except probes were not hydrolyzed (Prince et al. 1998). For experiments analyzing bmp3 expression, embryos were either manually de-yolked and flat-mounted under a coverslip in $70 \%$ glycerol or embedded in paraffin wax, sectioned, and mounted under a coverslip using Dibutylphthalate Polystyrene Xylene (DPX) mountant. Flat-mounts and sections were imaged on a Zeiss Axiolmager Z1 compound microscope with Axiocam HR digital camera. For experiments analyzing $t b x 5 a$, alhd1a2, vax2 and alhd1a3 expression, embryos were mounted in $3 \%$ methyl-cellulose on a spot plate and imaged with an Olympus SZX12 stereomicroscope and Qimaging micropublisher camera.

\section{Immunofluorescence}

For laminin immunofluorescence, embryos were fixed n 4\% PFA for two hours at RT. Embryos were permeabilized with $10 \mathrm{mg} / \mathrm{ml}$ Proteinase $\mathrm{K}$ for 30 minutes. After permeabilization, embryos were blocked for at least 1 hour at RT using 5\% normal goat serum and $2 \%$ bovine serum albumin (BSA) in PBST. Embryos were incubated $\mathrm{O} / \mathrm{N}$ in rabbit anti-laminin primary antibody diluted in block (1:200, L-9393, Sigma-Aldrich). After washing, embryos were incubated for 2 hours at RT in goat anti-rabbit Alexa Fluor 555 secondary antibody (1:1000, A32732, Invitrogen).

For Phosphorylated Histone H3 immunofluorescence, embryos were fixed in 4\% PFA for wo hours at RT. Embryos were permeabilized in ice-cold acetone for 7 minutes at $-20^{\circ} \mathrm{C}$. After permeabilization, embryos were incubated in $10 \mathrm{mM}$ ctric acid at $95 \mathrm{C}$ for 10 minutes for antigen retrieval. Embryos were then blocked in 3\% BSA in $0.5 \%$ TritonX-100 in PBS for 1 hour at RT. Embryos were then incubated in rabbit antiphospho-Histone $\mathrm{H} 3$ antibody $\left(1: 1000\right.$, ab183626, Abcam) $\mathrm{O} / \mathrm{N}$ at $4^{\circ} \mathrm{C}$, washed, and incubated in goat anti-rabbit Alexa Fluor 488 secondary antibody (1:1000, A32732, Invitrogen) plus TO-PRO-3 (1:1000, T3605, Invitrogen) for 2 hours at RT.

For cleaved Caspase 3 immunofluorescence, embryos were fixed in 4\% PFA for two hours at RT. Embryos were permeabilized with ice-cold acetone for 7 minutes at $-20^{\circ} \mathrm{C}$. After permeabilization, embryos were blocked in $5 \%$ goat serum (GS) in PBSDTT (PBST $+1 \%$ DMSO $+0.1 \%$ TritonX-100) for 2 hours at RT. Embryos were then incubated in rabbit anti-cleaved-Caspase3 $(1: 400,559565$, BDBiosciences) O/N at 
$4^{\circ} \mathrm{C}$, washed, and incubated in goat anti-rabbit Alexa Fluor 488 secondary antibody (1:1000, A32732, Invitrogen) plus TO-PRO-3 (1:1000, T3605, Invitrogen) for 2 hours at RT.

For phosphorylated Smad3 (pSmad3) immunofluorescence, embryos were fixed overnight at $4^{\circ} \mathrm{C}$. Embryos were permeabilized with $10 \mathrm{mg} / \mathrm{ml}$ Proteinase $\mathrm{K}$ for 5 minutes After permeabilization, embryos were incubated in $4 \%$ BSA in $0.15 \%$ TritonX-100 in PBS. Embryos were incubated in rabbit anti-pSmad3 primary antibody $\left(1: 200\right.$, ab59203, Abcam) $\mathrm{O} / \mathrm{N}$ at $4^{\circ} \mathrm{C}$, washed, and incubated in goat anti-rabbit Alexa Fluor 488 or 568 secondary antibody $0 / \mathrm{N}$ at $4^{\circ} \mathrm{C}(1: 1000, A 32732$, Invitrogen).

After immunofluorescence was performed, embryos were washed and passed through a 30,50 , and $70 \%$ glycerol series. Eyes and the surrounding head mesenchyme were dissected from whole embryos and mounted in $70 \%$ glycerol on a glass slide. All immunofluorescence experiments were imaged using a Zeiss Axiolmager Z1, Zeiss LSM700 laser scanning confocal microscope.

\section{Pharmacological inhibition of TGF-b signaling}

To inhibit TGF-b signaling, Specific Inhibitor of Smad3 (SIS3) was used (566405-1MG, Calbiochem). The structure and activity of SIS3 has been described elsewhere (Jinnin et al. 2006). $1 \mathrm{mg}$ of SIS3 was resuspended in DMSO to a stock concentration of $3 \mathrm{mM}$, aliquoted, and stored at $-20^{\circ} \mathrm{C}$. Prior to treatment, the stock was mixed with pre-warmed embryo media to a final concentration of $12 \mathrm{mM}$. For controls, an equivalent volume of DMSO alone was added to pre-warmed embryo media. Embryos were stagematched at $10 \mathrm{hpf}$. At $24 \mathrm{hpf}$, embryos were de-chorionated, transferred to $35 \mathrm{~mm}$ petri dishes in groups of 15 embryos per dish, and $5 \mathrm{~mL}$ of SIS3- or DMSO-treated media was added to the appropriate dishes. Embryos were incubated for six hours at $28.5^{\circ} \mathrm{C}$ until $30 \mathrm{hpf}$. The media was then removed, embryos were washed 3 times with fresh embryo media, and all dishes were returned to $28.5^{\circ} \mathrm{C}$. Embryo media was changed at $48 \mathrm{hpf}$, and embryos were fixed at $72 \mathrm{hpf}$ for 2 hours at RT with 4\% PFA. Embryos were then used for laminin immunofluorescence. Eyes from these embryos were then dissected, mounted on glass slides in 70\% glycerol, and imaged using a Zeiss Axiolmager Z1, Zeiss LSM700 laser scanning confocal microscope.

\section{Micro-computed tomography (micro-CT) of adult zebrafish}

18-month-old wildtype $(\mathrm{n}=5)$ and $\mathrm{bmp3}^{-/-}(\mathrm{n}=5)$ zebrafish were scanned using MILabs $\mu \mathrm{CT}$ at the School of Dentistry, University of Alberta. Zebrafish were fixed at 4\% PFA for $24 \mathrm{~h}$. The parameters for scanning and reconstruction were conducted as previously described (Miyashita et al. 2020). AVIZO (Life Technologies) software was used to visualize and quantify premaxilla, maxilla, and mandible length. Landmarks used to quantify the midfacial region are demonstrated in Figure S7.

\section{Alcian blue staining of larval zebrafish}

Alcian blue staining of $7 \mathrm{dpf}$ larval zebrafish was performed as previously described, except Alizarin red staining was not performed (Walker and Kimmel 2007). Briefly, larval zebrafish were euthanized using a 
$4 \%$ dilution of $0.4 \%$ TMS and fixed in 1\% PFA for one hour at RT. Embryos were washed for 10 minutes in $10 \mathrm{mM} \mathrm{MgCl}_{2}$ buffered with $100 \mathrm{mM}$ Tris pH 7.5, and then stained overnight in $0.04 \%$ Alcian blue staining solution. Embryos were removed from staining solution, rehydrated, bleached for 10 minutes in $3 \%$ $\mathrm{H}_{2} \mathrm{O}_{2} / 0.5 \% \mathrm{KOH}$, and de-stained with two 10 -minute washes of $25 \% \mathrm{Glycerol} / 0.1 \% \mathrm{KOH}$ and two 10 minute washes of $50 \%$ Glycerol $/ 0.1 \% \mathrm{KOH}$. Embryos were then transferred to $70 \%$ glycerol and mounted on glass slides for imaging. Images were taken using a Zeiss Axiolmager Z1 compound microscope with Axiocam HR digital camera.

Statistical analysis

Statistical significance was determined using a two-proportion z-test (Figure 5) or an unpaired t-test

(Figure 5,6 , and 7). Quantitative results are presented as percentage or mean \pm S.E.

\section{Declarations}

Acknowledgements- The authors are grateful to Science Animal Support Services for zebrafish animal care and facility maintenance

Conflict of Interest Statement- The authors declare no competing conflicts of interest.

Funding - This works was funded by the Women and Children's Health Research Institute (UOFAB WCHRIIG 2879) and the National Science and Engineering Research Council of Canada (NSERC RGPIN2016-04682)

Conflicts of Interest - The authors declare no conflicts of interest.

Availability of data and material - The data that support the findings of this study are available from the corresponding author upon reasonable request.

Code availability - Not applicable.

Ethics approval - Embryonic, larval, and adult zebrafish were cared for according to guidelines set by the Canadian Council of Animal Care and protocols were approved by the University of Alberta's Animal Care and Use Committee (Protocol \#427).

Consent to participate - Not applicable.

Consent to publish - Not applicable.

\section{References}


Abouzeid H, Boisset G, Favez T, et al (2011) Mutations in the SPARC-related modular calcium-binding protein 1 gene, SMOC1, cause waardenburg anophthalmia syndrome. Am J Hum Genet 88:92-98

Adler R, Belecky-Adams TL (2002) The role of bone morphogenetic proteins in the differentiation of the ventral optic cup. Development 129:3161 - 3171

Allendorph GP, Isaacs MJ, Kawakami Y, et al (2007) BMP-3 and BMP-6 Structures Illuminate the Nature of Binding Specificity with Receptors,. Biochemistry 46:12238-12247. https://doi.org/10.1021/bi700907k

ALSomiry AS, Gregory-Evans CY, Gregory-Evans K (2019) An update on the genetics of ocular coloboma. Hum Genet 138:865-880. https://doi.org/10.1007/s00439-019-02019-3

Asai-Coakwell M, French CR, Berry KM, et al (2007) GDF6, a novel locus for a spectrum of ocular developmental anomalies. Am J Hum Genet 80:306-315

Asai-Coakwell M, French CR, Ye M, et al (2009) Incomplete penetrance and phenotypic variability characterize Gdf6-attributable oculo-skeletal phenotypes. Hum Mol Genet 18:1110-1121. https://doi.org/10.1093/hmg/ddp008

Bahamonde ME, Lyons KM (2001) BMP3: To Be or Not To Be a BMP. JBJS 83: BJS 83: S56-S62

Bajpai R, Chen DA, Rada-Iglesias A, et al (2010) CHD7 cooperates with PBAF to control multipotent neural crest formation. Nature 463:958-962. https://doi.org/10.1038/nature08733

Bakrania P, Efthymiou M, Klein JC, et al (2008) Mutations in BMP4 cause eye, brain, and digit developmental anomalies: overlap between the BMP4 and hedgehog signaling pathways. Am J Hum Genet 82:304-319

Bassing $\mathrm{CH}$, Howe DJ, Segarini PR, et al (1994) A single heteromeric receptor complex is sufficient to mediate biological effects of transforming growth factor-beta ligands. J Biol Chem 269:14861-14864. https://doi.org/https://doi.org/10.1016/S0021-9258(17)36543-2

Beaulieu CL, Majewski J, Schwartzentruber J, et al (2014) FORGE Canada Consortium: Outcomes of a 2Year National Rare-Disease Gene-Discovery Project. Am J Hum Genet 94:809-817. https://doi.org/10.1016/j.ajhg.2014.05.003

Beby F, Commeaux C, Bozon M, et al (2007) New Phenotype Associated With an Arg116Cys Mutation in the CRYAA Gene: Nuclear Cataract, Iris Coloboma, and Microphthalmia. Arch Ophthalmol 125:213-216. https://doi.org/10.1001/archopht.125.2.213

Beleggia F, Li Y, Fan J, et al (2015) CRIM1 haploinsufficiency causes defects in eye development in human and mouse. Hum Mol Genet 24:2267-2273. https://doi.org/10.1093/hmg/ddu744 
Bernstein CS, Anderson MT, Gohel C, et al (2018) The cellular bases of choroid fissure formation and closure. Dev Biol 440:137-151. https://doi.org/https://doi.org/10.1016/j.ydbio.2018.05.010

Brady JP, Garland D, Duglas-Tabor Y, et al (1997) Targeted disruption of the mouse aA-crystallin gene induces cataract and cytoplasmic inclusion bodies containing the small heat shock protein aB-crystallin. Proc Natl Acad Sci 94:884 - 889. https://doi.org/10.1073/pnas.94.3.884

Bryan CD, Casey MA, Pfeiffer RL, et al (2020) Optic cup morphogenesis requires neural crest-mediated basement membrane assembly. Development 147:dev181420. https://doi.org/10.1242/dev.181420

Creuzet S, Vincent C, Couly G (2003) Neural crest derivatives in ocular and periocular structures. Int J Dev Biol 49:161-171

Daluiski A, Engstrand T, Bahamonde ME, et al (2001) Bone morphogenetic protein-3 is a negative regulator of bone density. Nat Genet 27:84-88. https://doi.org/10.1038/83810

Dee CT, Szymoniuk CR, Mills PED, Takahashi T (2012) Defective neural crest migration revealed by a Zebrafish model of Alx1-related frontonasal dysplasia. Hum Mol Genet 22:239-251.

https://doi.org/10.1093/hmg/dds423

Eckert P, Knickmeyer MD, Heermann S (2020) In Vivo Analysis of Optic Fissure Fusion in Zebrafish: Pioneer Cells, Basal Lamina, Hyaloid Vessels, and How Fissure Fusion is Affected by BMP. Int J Mol Sci $21: 2760$

Eve AMJ, Place ES, Smith JC (2017) Comparison of Zebrafish tmem88a mutant and morpholino knockdown phenotypes. PLoS One 12:e0172227

French CR, Erickson T, French D V, et al (2009) Gdf6a is required for the initiation of dorsal-ventral retinal patterning and lens development. Dev Biol 333:37-47

Gage PJ, Rhoades W, Prucka SK, Hjalt T (2005) Fate Maps of Neural Crest and Mesoderm in the Mammalian Eye. Invest Ophthalmol Vis Sci 46:4200-4208. https://doi.org/10.1167/iovs.05-0691

Gagnon JA, Valen E, Thyme SB, et al (2014) Efficient Mutagenesis by Cas9 Protein-Mediated Oligonucleotide Insertion and Large-Scale Assessment of Single-Guide RNAs. PLoS One 9:e98186

Gamer LW, Nove J, Levin M, Rosen V (2005) BMP-3 is a novel inhibitor of both activin and BMP-4 signaling in Xenopus embryos. Dev Biol 285:156-168.

https://doi.org/https://doi.org/10.1016/j.ydbio.2005.06.012

Gestri G, Bazin-Lopez N, Scholes C, Wilson SW (2018) Cell Behaviors during Closure of the Choroid Fissure in the Developing Eye. Front. Cell. Neurosci 12:42 
Gestri G, Osborne RJ, Wyatt AW, et al (2009) Reduced TFAP2A function causes variable optic fissure closure and retinal defects and sensitizes eye development to mutations in other morphogenetic regulators. Hum Genet 126:791-803. https://doi.org/10.1007/s00439-009-0730-x

Goebel EJ, Hart KN, McCoy JC, Thompson TB (2019) Structural biology of the TGF $\beta$ family. Exp Biol Med 244:1530-1546. https://doi.org/10.1177/1535370219880894

Gosse NJ, Baier H (2009) An essential role for Radar (Gdf6a) in inducing dorsal fate in the zebrafish retina. Proc Natl Acad Sci 106:2236 - 2241. https://doi.org/10.1073/pnas.0803202106

Graff JM, Bansal A, Melton DA (1996) Xenopus Mad Proteins Transduce Distinct Subsets of Signals for the TGF $\beta$ Superfamily. Cell 85:479-487. https://doi.org/https://doi.org/10.1016/S0092-8674(00)81249-0

Hanel ML, Hensey C (2006) Eye and neural defects associated with loss of GDF6. BMC Dev Biol 6:43. https://doi.org/10.1186/1471-213X-6-43

Hardy H, Prendergast JGD, Patel A, et al (2019) Detailed analysis of chick optic fissure closure reveals Netrin-1 as an essential mediator of epithelial fusion. Elife 8:e43877

Hero I (1990) Optic fissure closure in the normal cinnamon mouse. An ultrastructural study. Invest Ophthalmol Vis Sci 31:197-216

Hero I (1989) The optic fissure in the normal and microphthalmic mouse. Exp Eye Res 49:229-239. https://doi.org/https://doi.org/10.1016/0014-4835(89)90093-6

Hornby SJ, Dandona L, Jones RB, et al (2003) The familial contribution to non-syndromic ocular coloboma in south India. Br J Ophthalmol 87:336 - 340. https://doi.org/10.1136/bjo.87.3.336

Ittner LM, Wurdak H, Schwerdtfeger K, et al (2005) Compound developmental eye disorders following inactivation of TGF $\beta$ signaling in neural-crest stem cells. J Biol 4:11

James A, Lee C, Williams AM, et al (2016) The hyaloid vasculature facilitates basement membrane breakdown during choroid fissure closure in the zebrafish eye. Dev Biol 419:262-272. https://doi.org/https://doi.org/10.1016/j.ydbio.2016.09.008

Jinnin M, Ihn H, Tamaki K (2006) Characterization of SIS3, a Novel Specific Inhibitor of Smad3, and Its Effect on Transforming Growth Factor- $\beta 1$-Induced Extracellular Matrix Expression. Mol Pharmacol 69:597 - 607. https://doi.org/10.1124/mol.105.017483

Kagiyama Y, Gotouda N, Sakagami K, et al (2005) Extraocular dorsal signal affects the developmental fate of the optic vesicle and patterns the optic neuroepithelium. Dev Growth Differ 47:523-536. https://doi.org/10.1111/j.1440-169X.2005.00828.x 
Kim T-H, Goodman J, Anderson K V, Niswander L (2007) Phactr4 Regulates Neural Tube and Optic Fissure Closure by Controlling PP1-, Rb-, and E2F1-Regulated Cell-Cycle Progression. Dev Cell 13:87-102. https://doi.org/https://doi.org/10.1016/j.devcel.2007.04.018

Kimmel CB, Ballard WW, Kimmel SR, et al (1995) Stages of embryonic development of the zebrafish. Dev Dyn 203:253-310

Kimmel CB, Miller CT, Kruze G, et al (1998) The Shaping of Pharyngeal Cartilages during Early Development of the Zebrafish. Dev Biol 203:245-263.

https://doi.org/https://doi.org/10.1006/dbio.1998.9016

Knickmeyer MD, Mateo JL, Eckert P, et al (2020) TGF $\beta$-facilitated optic fissure fusion and the role of bone morphogenetic protein antagonism. Open Biol 8:170134. https://doi.org/10.1098/rsob.170134

Kwan KM, Otsuna $\mathrm{H}$, Kidokoro $\mathrm{H}$, et al (2012) A complex choreography of cell movements shapes the vertebrate eye. Development 139:359 - 372. https://doi.org/10.1242/dev.071407

Langenberg T, Kahana A, Wszalek JA, Halloran MC (2008) The eye organizes neural crest cell migration. Dev Dyn 237:1645-1652. https://doi.org/10.1002/dvdy.21577

Laurie KJ, Dave A, Straga T, et al (2013) Identification of a Novel Oligomerization Disrupting Mutation in CRYAA Associated with Congenital Cataract in a South Australian Family. Hum Mutat 34:435-438. https://doi.org/https://doi.org/10.1002/humu.22260

Lee J, Lee B-K, Gross JM (2013) Bcl6a function is required during optic cup formation to prevent p53dependent apoptosis and colobomata. Hum Mol Genet 22:3568-3582.

https://doi.org/10.1093/hmg/ddt211

Levitt M (1978) Conformational preferences of amino acids in globular proteins. Biochemistry 17:42774285. https://doi.org/10.1021/bi00613a026

Litt M, Kramer P, LaMorticella DM, et al (1998) Autosomal Dominant Congenital Cataract Associated with a Missense Mutation in the Human Alpha Crystallin Gene CRYAA. Hum Mol Genet 7:471-474. https://doi.org/10.1093/hmg/7.3.471

Little SC, Mullins MC (2006) Extracellular modulation of BMP activity in patterning the dorsoventral axis. Birth Defects Res Part C Embryo Today Rev 78:224-242. https://doi.org/https://doi.org/10.1002/bdrc.20079

Liu F, Hata A, Baker JC, et al (1996) A human Mad protein acting as a BMP-regulated transcriptional activator. Nature 381:620-623. https://doi.org/10.1038/381620a0

Lo RS, Chen YG, Shi Y, et al (1998) The L3 loop: a structural motif determining specific interactions between SMAD proteins and TGF-beta receptors. EMBO J 17:996-1005. 
Lupo G, Gestri G, O’Brien M, et al (2011) Retinoic acid receptor signaling regulates choroid fissure closure through independent mechanisms in the ventral optic cup and periocular mesenchyme. Proc Natl Acad Sci USA 108:8698-8703. https://doi.org/10.1073/pnas.1103802108

Mackay DS, Andley UP, Shiels A (2003) Cell death triggered by a novel mutation in the alphaA-crystallin gene underlies autosomal dominant cataract linked to chromosome 21q. Eur J Hum Genet 11:784-793. https://doi.org/10.1038/sj.ejhg.5201046

McMahon C, Gestri G, Wilson SW, Link BA (2009) Lmx1b is essential for survival of periocular mesenchymal cells and influences Fgf-mediated retinal patterning in zebrafish. Dev Biol 332:287-298. https://doi.org/10.1016/j.ydbio.2009.05.577

Melo, F., Devos, D., Depiereux, E., Feytmans E (1997) ANOLEA: a www server to assess protein structures. Intell Syst Mol Biol 97:110-113

Miyashita T, Baddam P, Smeeton J, et al (2020) nkx3.2 mutant zebrafish accommodate jaw joint loss through a phenocopy of the head shapes of Paleozoic jawless fish. J Exp Biol 223:. https://doi.org/10.1242/jeb.216945

Miyazono K, Ten Dijke P, Heldin C-H (2000) TGF- $\beta$ signaling by Smad proteins. Academic Press, pp 115157

Morcillo J, Martínez-Morales JR, Trousse F, et al (2006) Proper patterning of the optic fissure requires the sequential activity of BMP7 and SHH. Development 133:3179 LP - 3190.

https://doi.org/10.1242/dev.02493

Morrison D, FitzPatrick D, Hanson I, et al (2002) National study of microphthalmia, anophthalmia, and coloboma (MAC) in Scotland: investigation of genetic aetiology. J Med Genet 39:16 - 22. https://doi.org/10.1136/jmg.39.1.16

Mueller RL, Huang C, Ho RK (2010) Spatio-temporal regulation of Wnt and retinoic acid signaling by tbx16/spadetail during zebrafish mesoderm differentiation. BMC Genomics 11:492. https://doi.org/10.1186/1471-2164-11-492

Noh H, Lee H, Park E, Park S (2016) Proper closure of the optic fissure requires ephrin A5-EphB2-JNK signaling. Development 143:461-472. https://doi.org/10.1242/dev.129478

Okada I, Hamanoue H, Terada K, et al (2011) SMOC1 Is Essential for Ocular and Limb Development in Humans and Mice. Am J Hum Genet 88:30-41. https://doi.org/10.1016/j.ajhg.2010.11.012

Patel A, Sowden JC (2019) Genes and pathways in optic fissure closure. Semin Cell Dev Biol 91:55-65. https://doi.org/https://doi.org/10.1016/j.semcdb.2017.10.010 
Peters MA, Cepko CL (2002) The Dorsal-Ventral Axis of the Neural Retina Is Divided into Multiple Domains of Restricted Gene Expression Which Exhibit Features of Lineage Compartments. Dev Biol 251:59-73. https://doi.org/https://doi.org/10.1006/dbio.2002.0791

Pfirrmann T, Emmerich D, Ruokonen P, et al (2015) Molecular mechanism of CHRDL1-mediated X-linked megalocornea in humans and in Xenopus model. Hum Mol Genet 24:3119-3132.

https://doi.org/10.1093/hmg/ddv063

Pras E, Frydman M, Levy-Nissenbaum E, et al (2000) A Nonsense Mutation (W9X) in CRYAA Causes Autosomal Recessive Cataract in an Inbred Jewish Persian Family. Invest Ophthalmol Vis Sci 41:35113515

Prince VE, Moens CB, Kimmel CB, Ho RK (1998) Zebrafish hox genes: expression in the hindbrain region of wild-type and mutants of the segmentation gene, valentino. Development 125:393 LP - 406

Prokudin I, Simons C, Grigg JR, et al (2014) Exome sequencing in developmental eye disease leads to identification of causal variants in GJA8, CRYGC, PAX6 and CYP1B1. Eur J Hum Genet 22:907-915. https://doi.org/10.1038/ejhg.2013.268

Reis LM, Tyler RC, Schilter KF, et al (2011) BMP4 loss-of-function mutations in developmental eye disorders including SHORT syndrome. Hum Genet 130:495-504. https://doi.org/10.1007/s00439-0110968-y

Richter L, Flodman P, Barria von-Bischhoffshausen F, et al (2008) Clinical variability of autosomal dominant cataract, microcornea and corneal opacity and novel mutation in the alpha A crystallin gene (CRYAA). Am J Med Genet Part A 146A:833-842. https://doi.org/https://doi.org/10.1002/ajmg.a.32236

Rochard L, Monica SD, Ling ITC, et al (2016) Roles of Wnt pathway genes wls, wnt9a, wnt5b, frzb and gpc4 in regulating convergent-extension during zebrafish palate morphogenesis. Development 143:2541-2547. https://doi.org/10.1242/dev.137000

Sakuta H, Suzuki R, Takahashi H, et al (2001) Ventroptin: A BMP-4 Antagonist Expressed in a DoubleGradient Pattern in the Retina. Science (80-) 293:111 LP - 115.

https://doi.org/10.1126/science.1058379

Schmitt EA, Dowling JE (1994) Early-eye morphogenesis in the zebrafish, Brachydanio rerio. J Comp Neurol 344:532-542. https://doi.org/10.1002/cne.903440404

Schoenebeck JJ, Hutchinson SA, Byers A, et al (2012) Variation of BMP3 Contributes to Dog Breed Skull Diversity. PLOS Genet 8:e1002849

Sedykh I, Yoon B, Roberson L, et al (2017) Zebrafish zic2 controls formation of periocular neural crest and choroid fissure morphogenesis. Dev Biol 429:92-104. https://doi.org/10.1016/j.ydbio.2017.07.003 
Sghari S, Gunhaga L (2018) Temporal Requirement of Mab21/2 During Eye Development in Chick Reveals Stage-Dependent Functions for Retinogenesis. Invest Ophthalmol Vis Sci 59:3869-3878. https://doi.org/10.1167/iovs.18-24236

Shah SP, Taylor AE, Sowden JC, et al (2012) Anophthalmos, Microphthalmos, and Coloboma in the United Kingdom: Clinical Features, Results of Investigations, and Early Management. Ophthalmology 119:362368. https://doi.org/10.1016/j.ophtha.2011.07.039

Siebert JR, Graham Jr JM, MacDonald C (1985) Pathologic features of the CHARGE association: support for involvement of the neural crest. Teratology 31:331-336

Skarie JM, Link BA (2009) FoxC1 Is Essential for Vascular Basement Membrane Integrity and Hyaloid Vessel Morphogenesis. Invest Ophthalmol Vis Sci 50:5026-5034. https://doi.org/10.1167/iovs.09-3447

Stewart A, Guan H, Yang K (2010) BMP-3 promotes mesenchymal stem cell proliferation through the TGFß/activin signaling pathway. J Cell Physiol 223:658-666. https://doi.org/10.1002/jcp.22064

Sun Z, Zhou Q, Li H, et al (2017) Mutations in crystallin genes result in congenital cataract associated with other ocular abnormalities. Mol Vis 23:977-986

Suzuki S, Marazita ML, Cooper ME, et al (2009) Mutations in BMP4 Are Associated with Subepithelial, Microform, and Overt Cleft Lip. Am J Hum Genet 84:406-411.

https://doi.org/10.1016/j.ajhg.2009.02.002

Takamiya M, Stegmaier J, Kobitski AY, et al (2020) Pax6 organizes the anterior eye segment by guiding two distinct neural crest waves. PLoS Genet 16:. https://doi.org/10.1371/journal.pgen.1008774

Thisse C, Thisse B (2008) High-resolution in situ hybridization to whole-mount zebrafish embryos. Nat Protoc 3:59-69. https://doi.org/10.1038/nprot.2007.514

Trost A, Schroedl F, Lange S, et al (2013) Neural Crest Origin of Retinal and Choroidal Pericytes. Invest Ophthalmol Vis Sci 54:7910-7921. https://doi.org/10.1167/iovs.13-12946

Tsuji N, Kita K, Ozaki K, et al (2012) Organogenesis of mild ocular coloboma in FLS mice: Failure of basement membrane disintegration at optic fissure margins. Exp Eye Res 94:174-178. https://doi.org/https://doi.org/10.1016/j.exer.2011.12.004

Van Nostrand JL, Brady CA, Jung H, et al (2014) Inappropriate p53 activation during development induces features of CHARGE syndrome. Nature 514:228-232. https://doi.org/10.1038/nature13585

Viringipurampeer IA, Ferreira T, DeMaria S, et al (2012) Pax2 regulates a fadd-dependent molecular switch that drives tissue fusion during eye development. Hum Mol Genet 21:2357-2369.

https://doi.org/10.1093/hmg/dds056 
Walker M, Kimmel C (2007) A two-color acid-free cartilage and bone stain for zebrafish larvae. Biotech Histochem 82:23-28. https://doi.org/10.1080/10520290701333558

Williamson KA, FitzPatrick DR (2014) The genetic architecture of microphthalmia, anophthalmia and coloboma. Eur J Med Genet 57:369-380. https://doi.org/https://doi.org/10.1016/j.ejmg.2014.05.002

Wrana JL, Attisano L, Cárcamo J, et al (1992) TGF-beta signals through a heteromeric protein kinase receptor complex. Cell 71:1003-1014. https://doi.org/10.1016/0092-8674(92)90395-S

Wyatt AW, Osborne RJ, Stewart H, Ragge NK (2010) Bone morphogenetic protein 7 (BMP7) mutations are associated with variable ocular, brain, ear, palate, and skeletal anomalies. Hum Mutat 31:781-787. https://doi.org/https://doi.org/10.1002/humu.21280

Yan X, Atorf J, Ramos D, et al (2020) Mutation in Bmpr1b Leads to Optic Disc Coloboma and Ventral Retinal Gliosis in Mice. Invest Ophthalmol Vis Sci 61:44. https://doi.org/10.1167/iovs.61.2.44

Ye M, Berry-Wynne KM, Asai-Coakwell M, et al (2010) Mutation of the bone morphogenetic protein GDF3 causes ocular and skeletal anomalies. Hum Mol Genet 19:287-298.

https://doi.org/10.1093/hmg/ddp496

Yoon KH, Fox SC, Dicipulo R, et al (2020) Ocular coloboma: Genetic variants reveal a dynamic model of eye development. Am J Med Genet Part C Semin Med Genet n/a:590-610.

https://doi.org/10.1002/ajmg.c.31831

Zhang R, Huang H, Cao P, et al (2013) Sma- and Mad-related Protein 7 (Smad7) Is Required for Embryonic Eye Development in the Mouse *. J Biol Chem 288:10275-10285.

https://doi.org/10.1074/jbc.M112.416719

Zhang Y, Feng X-H, Wu R-Y, Derynck R (1996) Receptor-associated Mad homologues synergize as effectors of the TGF- $\beta$ response. Nature 383:168-172. https://doi.org/10.1038/383168a0

Zuber ME, Gestri G, Viczian AS, et al (2003) Specification of the vertebrate eye by a network of eye field transcription factors. Development 130:5155-5167

\section{Tables}

Table 1. A list of candidate genes identified from exome sequencing of patients from a pedigree of autosomal dominant coloboma and microphthalmia. Exome sequencing was conducted on four affected individuals and one unaffected individual. Exome sequencing data was analyzed to only include nonsynonymous variants that were not detected in the unaffected control individual or in control exome databases. A SIFT score closer to 0 indicates a variant that is potentially disease-causing, while a PolyPhen 2 score closer to 1 indicates a variant that is potentially disease-causing. 


\begin{tabular}{llll} 
Gene & Amino acid variant & SIFT score & PolyPhen2 score \\
\hline BMP3 & p.Ala470Pro & 0 & 1 \\
\hline VPS72 & p.Tyr89Cys & 0 & 0.992 \\
\hline NLRC3 & p.Arg51Trp & 0 & 0.998 \\
\hline SARM1 & p.Arg230Cys & 0 & 1 \\
\hline SEMA5B & p.Arg273His & 0 & 0.992 \\
\hline PDZD9 & p.Glu104* & $\mathrm{n} / \mathrm{a}$ & $\mathrm{n} / \mathrm{a}$ \\
\hline TTC3 & p.Pro1239Ser & $\mathrm{n} / \mathrm{a}$ & 0.999 \\
\hline ZNF658 & p.Pro657Leu & 0 & 0.999 \\
\hline MYH3 & p.Asp1178Asn & 0.01 & 0.994 \\
\hline CRYAA & p.Arg96Cys & 0 & 1
\end{tabular}

Table 2. BMP3 variants identified in this study are found at extremely low frequencies in genome databases. None of the variants are detected in unaffected control samples. The p.Ala470Pro variant is not detected in any genome databases, while the p.Phe450Tyr and p.Ser393Phe variants are found only at extremely low frequencies in genome databases. (CDS=coding sequence, $A A=$ amino acid, $\mathrm{NHLBI}=$ National Heart, Lung, and Blood Institute Exome Variant Server, 1KG=1000 Genomes Project, gnomAD=Genome Aggregation Database).

\begin{tabular}{llllll} 
CDS Change & AA Change & Controls & NHLBI & 1KG & gnomAD \\
\hline c.1178C $>$ T & p.Ser393Phe & $0 / 192$ & $0 / 6732$ & $0 / 5000$ & $1 / 76,069$ \\
\hline c.1349T $>$ A & p.Phe450Tyr & $0 / 268$ & $1 / 6732$ & $1 / 5000$ & $29 / 76,064$ \\
\hline c.1408G $>$ C & p.Ala470Pro & $0 / 257$ & $0 / 6732$ & $0 / 5000$ & $0 / 76,156$
\end{tabular}

Table 3. The BMP3 variants identified in this study are predicted to be deleterious to protein function. All three variants in this study are predicted to be damaging to protein function by SIFT, PolyPhen2, and MutationTaster with the exception of pPhe450Tyr, which is predicted by SIFT to be tolerated. (CDS=coding sequence, $A A=$ amino acid). 
CDS

Change
c. $1178 \mathrm{C}>\mathrm{T}$
p.Ser393Phe
0.00 (Not
Tolerated)
1.00 (Probably
Damaging)
0.999 (Disease
Causing)
c. $1349 \mathrm{~T}>\mathrm{A}$
p.Phe450Tyr
0.13 (Tolerated)
0.995 (Probably
Damaging)
0.999 (Disease
Causing)
c. $1408 \mathrm{G}>\mathrm{C}$
p.Ala470Pro
$0.00($ Not
Tolerated $)$
1.00 (Probably
Damaging)
0.999 (Disease
Causing)

\section{Figures}

A

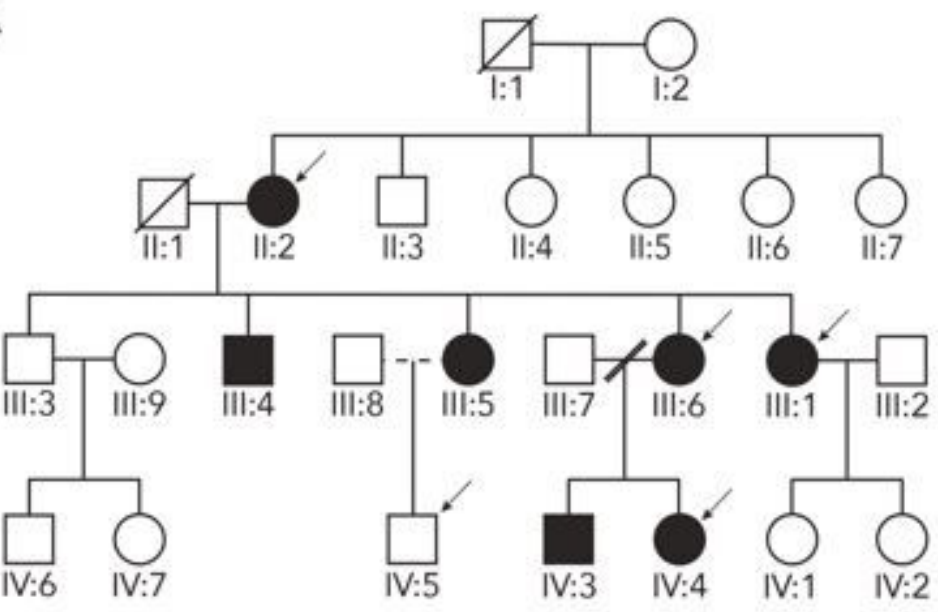

Whole exome

sequencing

7876 Variants

Not present in

48 Variants

In silico predictions, SNP databases

10 Variants

Model organism

expression

1 Variant

c. $1408 \mathrm{G}>\mathrm{C}$

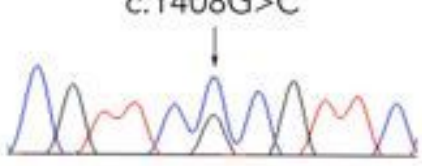

B
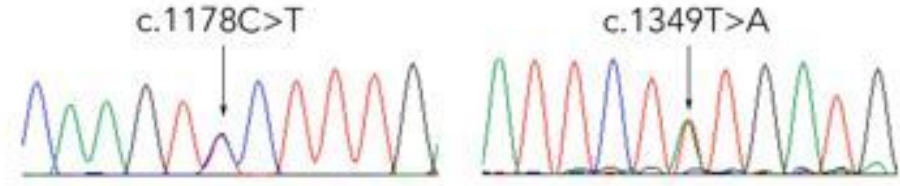

c. $1178 \mathrm{C}>\mathrm{T} \quad$ c. $1349 \mathrm{~T}>\mathrm{A} \quad$ c. $1408 \mathrm{G}>\mathrm{C}$

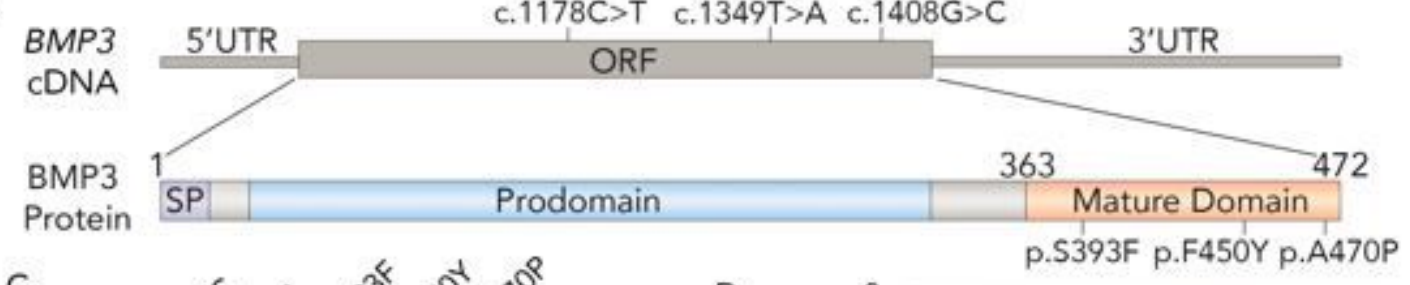

C

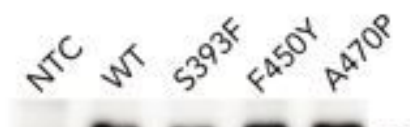

V5-lysate

V5-secreted

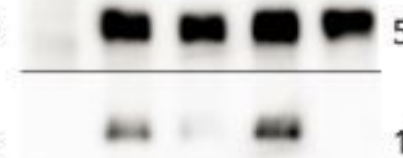

Tubulin

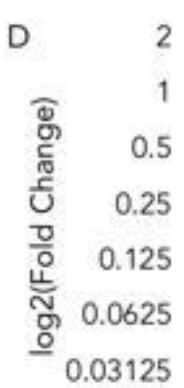

$53.5 \mathrm{kDa}$

$13.6 \mathrm{kDa}$

55 kDa

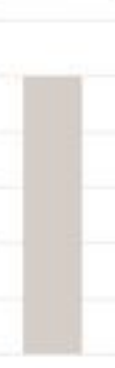

WT

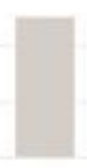

S393F

F450Y A470P 
BMP3 missense mutations identified in patients with ocular coloboma and microphthalmia. A) Fourgeneration pedigree of a family with autosomal dominant coloboma and/or microphthalmia. Whole exome sequencing (WES) was performed on DNA samples from specified individuals (arrows), and identified variants were filtered according to standardized criteria (flow chart, right). WES identified a variant in BMP3 (c.1408G $>$ C), and two additional variants were identified from Sanger sequencing of an independent cohort of unrelated patients (c.1178C $>T$ and c.1349T $>A$ ). Below, chromatograms showing DNA sequence variants indicated by their base change within the coding sequence of BMP3. B) Schematic of the BMP3 CDNA and protein, with the position of each nucleotide variant and resulting amino acid substitution indicated. ( $\mathrm{SP}=$ signal peptide, $\mathrm{ORF}=$ open reading frame). $\mathrm{C}$ ) Immunoblots for V5tagged BMP3 wildtype and variants. Cos-7 cells were transfected with equal amounts of plasmid DNA encoding V5-tagged BMP3 proteins, then immunoblots were performed on protein extracted from either the culture media or cell lysates. Immunoblots performed on cell lysates (top, V5-lysate) show that all BMP3 proteins are made in approximately equal amounts, but those performed on protein extracted from the culture media (middle, V5-secreted) demonstrate that the S393F and A470P variants, but not F450Y, result in reduced secretion of BMP3 protein. D) Quantification of three independent immunoblots. Band intensity for V5-secreted WT, S393F, F450Y, and A470P was measured using Image J software and the fold change in intensity for S393F, F450Y, and A470P was calculated relative to WT. Quantification indicates that the bands for V5-secreted S393F, F450Y, and A470P variants display a 0.18, 1.71, and 0.04fold change in intensity relative to WT. Results are representative of three independent experiments and immunoblots. (NTC=no transfection control, kDa=kilodaltons). 
A

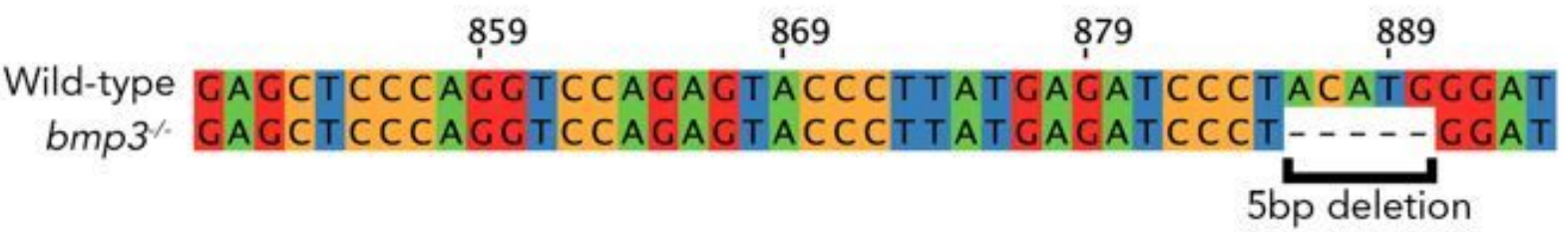

\begin{tabular}{c|c|c|c|} 
Wild-type & SP & Prodomain & Mature Domain \\
bmp3 $\%$ & SP & Prodomain &
\end{tabular}

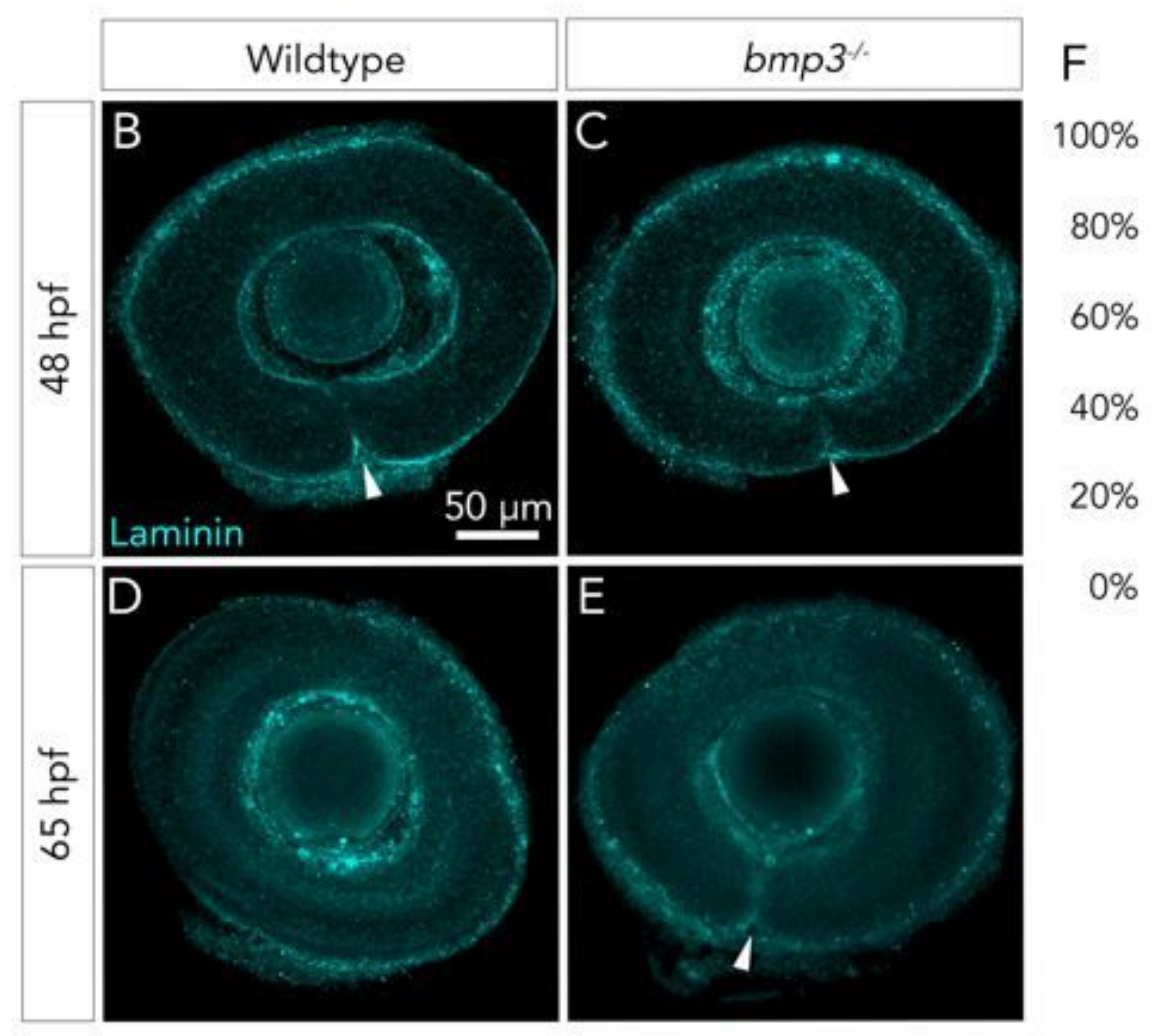

F
$100 \%$
$80 \%$
$60 \%$
$40 \%$
$20 \%$
$0 \%$

\section{Figure 2}

Zebrafish with mutations in bmp3 exhibit delayed fissure closure. A) Partial coding sequence alignment of wild type and bmp3ua1020 mutant zebrafish (top) and schematics of each resulting protein (bottom). A 5bp deletion (c.887_891del) was generated with CRISPR-Cas9, resulting in a frameshift and subsequent premature stop codon ( $\mathrm{p}$.Thr96GlyfsTer2). The resulting truncated protein lacks the entire TGF- $\beta$ mature domain. (SP=Signal peptide) (B-E) Larvae from a bmp3-/- heterozygous incross were fixed at 48 or $65 \mathrm{hpf}$ and laminin immunofluorescence was conducted to visualize the extracellular matrix. Eyes were manually dissected and mounted in pairs, then imaged on a confocal microscope. Both wild type and bmp3 mutant larvae at $48 \mathrm{hpf}(\mathrm{B}, \mathrm{C})$ show fissure lobes in apposition but incomplete fissure closure (indicated by arrows). In contrast, at $65 \mathrm{hpf}(\mathrm{D}, \mathrm{E})$ wild type larvae have completed fissure closure, while 23/130 bmp3 mutants show bilateral open fissures (arrowheads). F) Quantification of fissure phenotypes in $\mathrm{B}-\mathrm{E}$, showing $18 \%$ bilateral open fissures in bmp3 mutant larvae at $65 \mathrm{hpf}$. (WT=Wild-type, Open=0pen choroid fissures, Closed=Closed choroid fissures). 

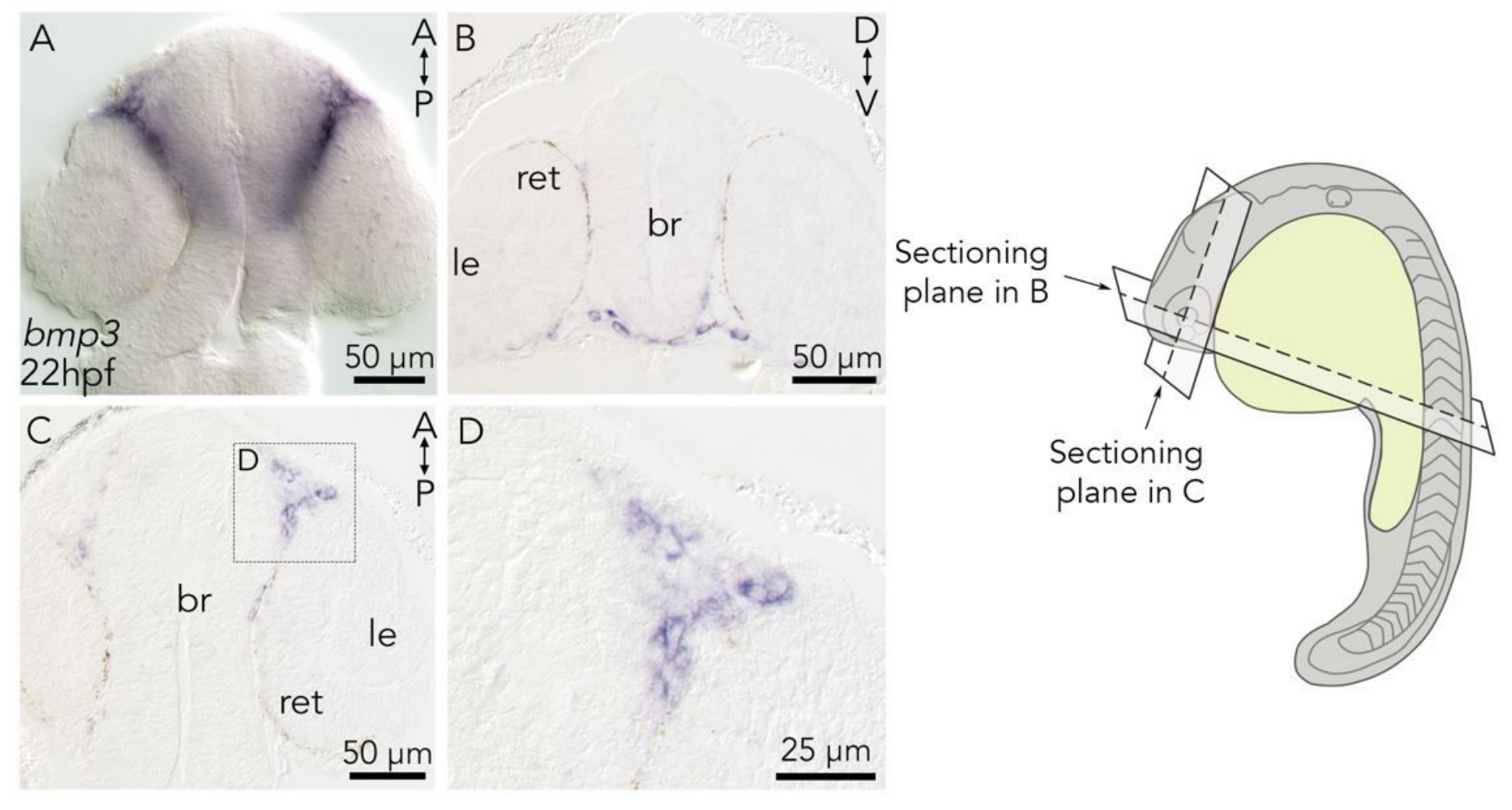

\section{Figure 3}

bmp3 is expressed in the periocular mesenchyme during ocular morphogenesis. Whole mount in situ hybridization was conducted on wildtype embryos to assess the localization of bmp3 mRNA during early ocular morphogenesis. A) Dorsal view of dissected and flat mounted embryo at $22 \mathrm{hpf}$. bmp3 expression is localized head mesenchyme between the retina and the forebrain. B-D) Embryos were embedded in paraffin wax after in situ hybridization, then sectioned and mounted on slides. Coronal sections through the middle of the optic cup (B) show bmp3 expression in a single cell-thick layer in the head mesenchyme directly ventral to the eye. Transverse sections (C, D) show that bmp3 transcript is specifically expressed in the head mesenchyme between the forebrain and the retina. The box in $C$ indicates the area magnified in $D$. ( $A=$ anterior, $P=$ posterior, $D=$ dorsal, $V=$ ventral, le=lens, br=brain, ret=retina). 


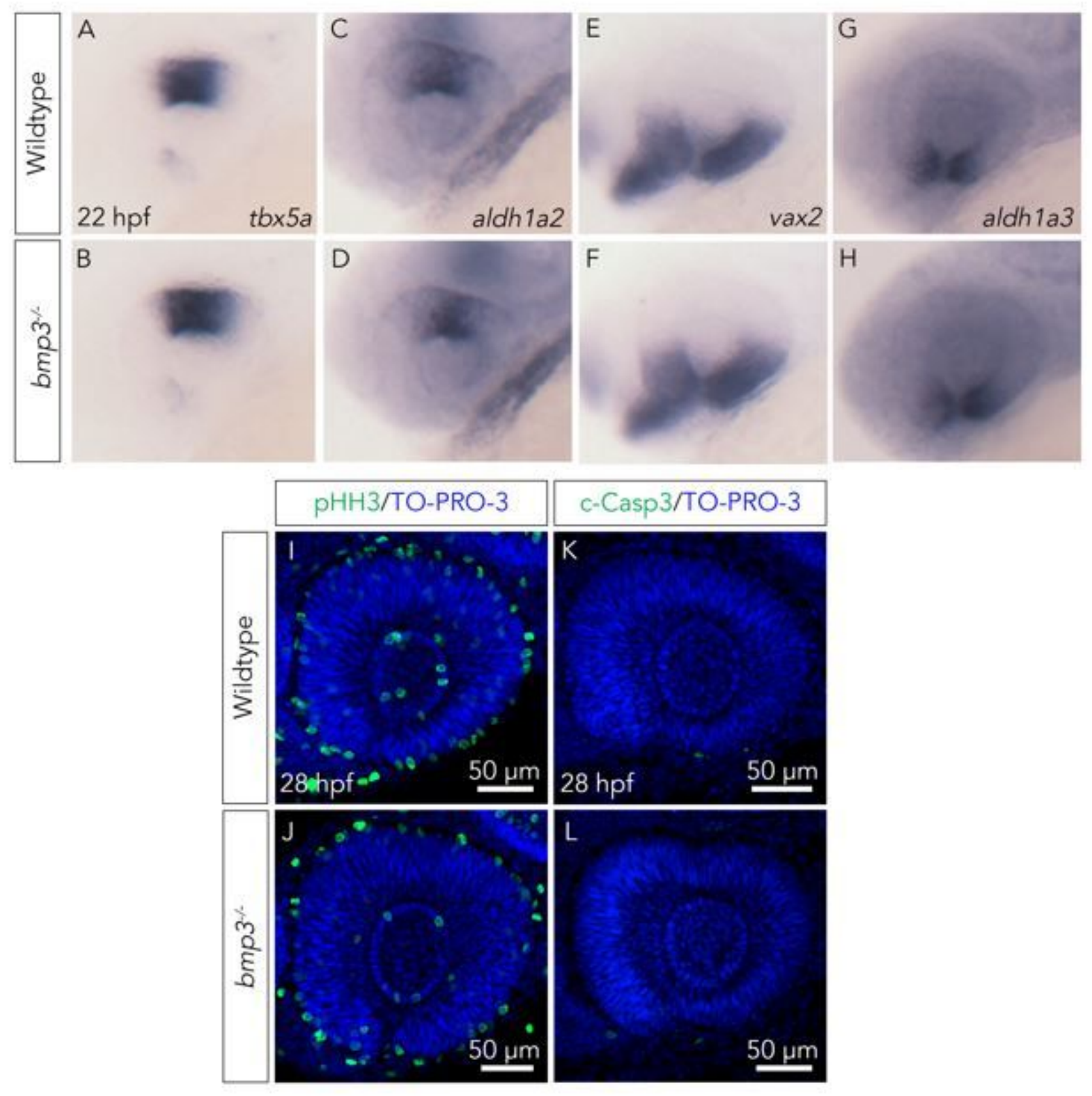

\section{Figure 4}

bmp3 does not regulate dorsal-ventral patterning, cell proliferation or apoptosis of the optic cup. A-H) To assess dorsal-ventral patterning of the optic cup in wildtype and bmp3 mutants, whole mount in situ hybridization was conducted on $22 \mathrm{hpf}$ embryos from a bmp3+/-incross, using probes for transcripts that provide a readout of dorsal (tbx5a, aldh1a2; A-D) and ventral (vax2, aldh1a3; E-H) optic cup identity. The expression of dorsal and ventral genes in bmp3-/- mutants $(B, D, F$ and $H)$ is comparable in both intensity and domain to wildtype embryos (A, C, E and G). Embryos were imaged laterally in $3 \%$ methylcellulose on a dissecting microscope then used for genomic DNA extraction and genotyping ( $n=25$ /probe-genotype combination). I-L)To assess proliferation and apoptosis of the optic cup in wildtype and bmp3 mutants, phosphorylated Histone $\mathrm{H} 3$ and cleaved Caspase 3 immunofluorescence was conducted on wildtype and bmp3-/- mutant embryos at $28 \mathrm{hpf}$. There is no observable difference in proliferation between wildtype (I) and bmp3-/- mutant ( $\mathrm{J}$ ) embryos ( $\mathrm{n}=20 /$ genotype), nor is there an 
observable difference in the amount of apoptosis in the optic cup of wildtype (K) or bmp3-/- mutant (L) embryos ( $n=19 /$ genotype). (pHH3=phospho-Histone H3, c-Casp3=cleaved Caspase 3).
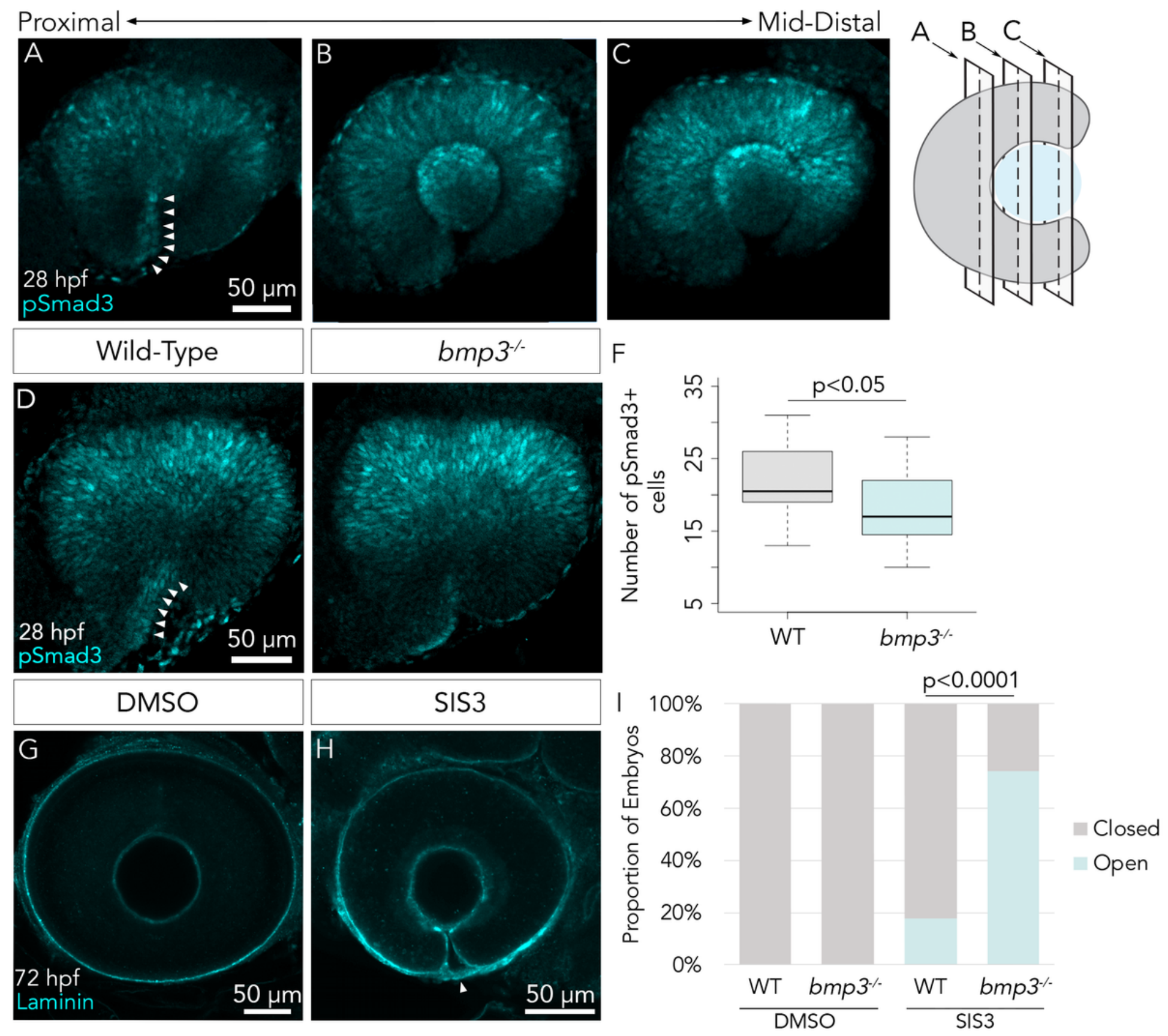

\section{Figure 5}

Smad3 phosphorylation is necessary for choroid fissure closure. A-C) pSmad3 immunofluorescence conducted on wildtype $28 \mathrm{hpf}$ embryos indicates that TGF- $\beta$ signaling is active in the nasal lobe of the proximal-ventral eye cup (A, arrowheads) but is largely absent in more distal regions of the choroid fissure (B-C). D-F) To assess Smad3 phosphorylation in bmp3 mutants, pSmad3 immunofluorescence was conducted on bmp3 mutants (E) and wildtype siblings (D) at $28 \mathrm{hpf}$. The number of pSmad3+ cells is reduced in a bmp3 mutants when compared to wildtype siblings (arrowheads, quantified in F). F) When quantified, there is a significant decrease in the number of pSmad3+ cells in bmp3 mutants $(18.3 \pm 1.09$ cells) when compared to wildtype (22.2 \pm 1.12 cells) (two-sample t-test, $t 38=2.49, p=0.0172)$ G-I) Wildtype 
and bmp3 mutant embryos were treated with a suboptimal dose $(12 \mu \mathrm{M})$ of SIS3 from $24-30 \mathrm{hpf}$, and the presence of open choroid fissures was assessed using laminin immunofluorescence. Both wildtype and bmp3-/- embryos display open choroid fissures when treated with SIS3 ( $\mathrm{H}$, arrowhead), compared to embryos treated with vehicle (DMSO) alone (G) (Wildtype embryos shown as an example) I) A significantly higher proportion of bmp3-/- mutant embryos have open choroid fissures compared to wildtype when treated with SIS3 (two-proportion z-test, $z=-4.32, p<0.0001$ ), suggesting that Bmp3 and Smad3 function in the same pathway. Results are representative of three independent experiments. (WT=Wildtype, Open=Open choroid fissure, Closed=Closed choroid fissure).
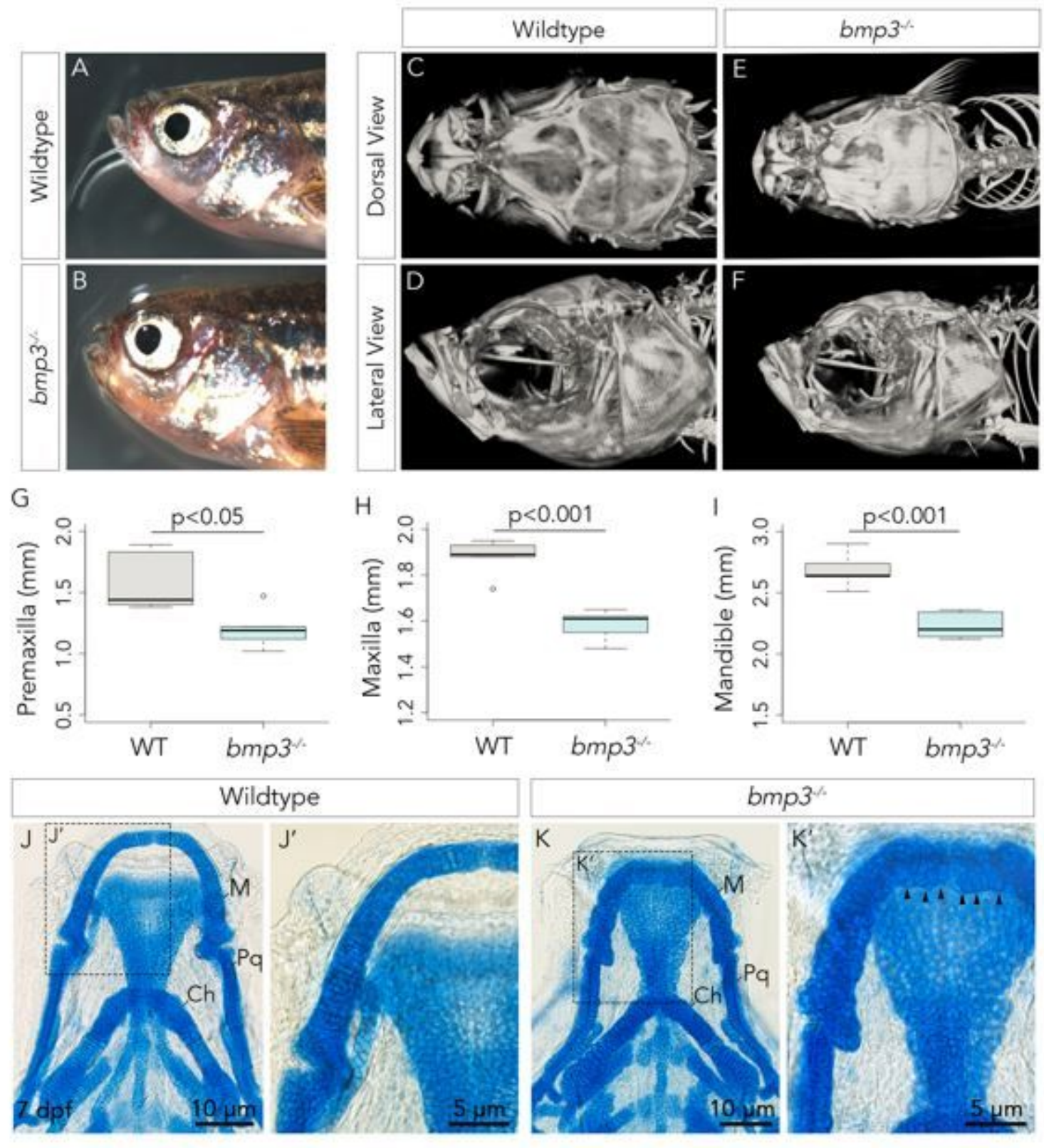

Figure 6

bmp3-/- mutants have midface hypoplasia. Dissecting microscope images (A, B) and Micro-CT analysis (C-F) of 18-month-old wildtype (A, C, D) and bmp3-/- (B, E, F) zebrafish demonstrate significantly shorter premaxilla $(G)$, maxilla $(H)$ and mandible $(I)$ in the mutant fish $(n=5)$ when compared to wildtype siblings 
$(\mathrm{n}=5)$ (two sample t-test, $\mathrm{t} 8=2.85,6.22$, and 5.50, $\mathrm{p}=0.000255,0.0214$, and 0.000573) (WT=wildtype). J-K') Alcian blue staining of craniofacial cartilage in $7 \mathrm{dpf}$ larvae indicates that $40 \%(8 / 20)$ of bmp3-/- mutants $(\mathrm{K})$ have disrupted jaw cartilage formation when compared to wildtype $(\mathrm{J})$ larvae $(0 / 20)$. The overall anatomy of Meckel's cartilage, the palatoquadrate, and the ceratohyal ( $\mathrm{J}$ and $\mathrm{K}$ ) as well as chondrocyte organization and morphology $\left(J^{\prime}-K^{\prime}\right.$, arrowheads) are abnormal in a subset of mutants $(n=8 / 20)$ when compared to wildtype controls $(n=0 / 20)(M=$ Meckel's cartilage, $P q=P a l a t o q u a d r a t e, C h=C e r a t o h y a l)$.

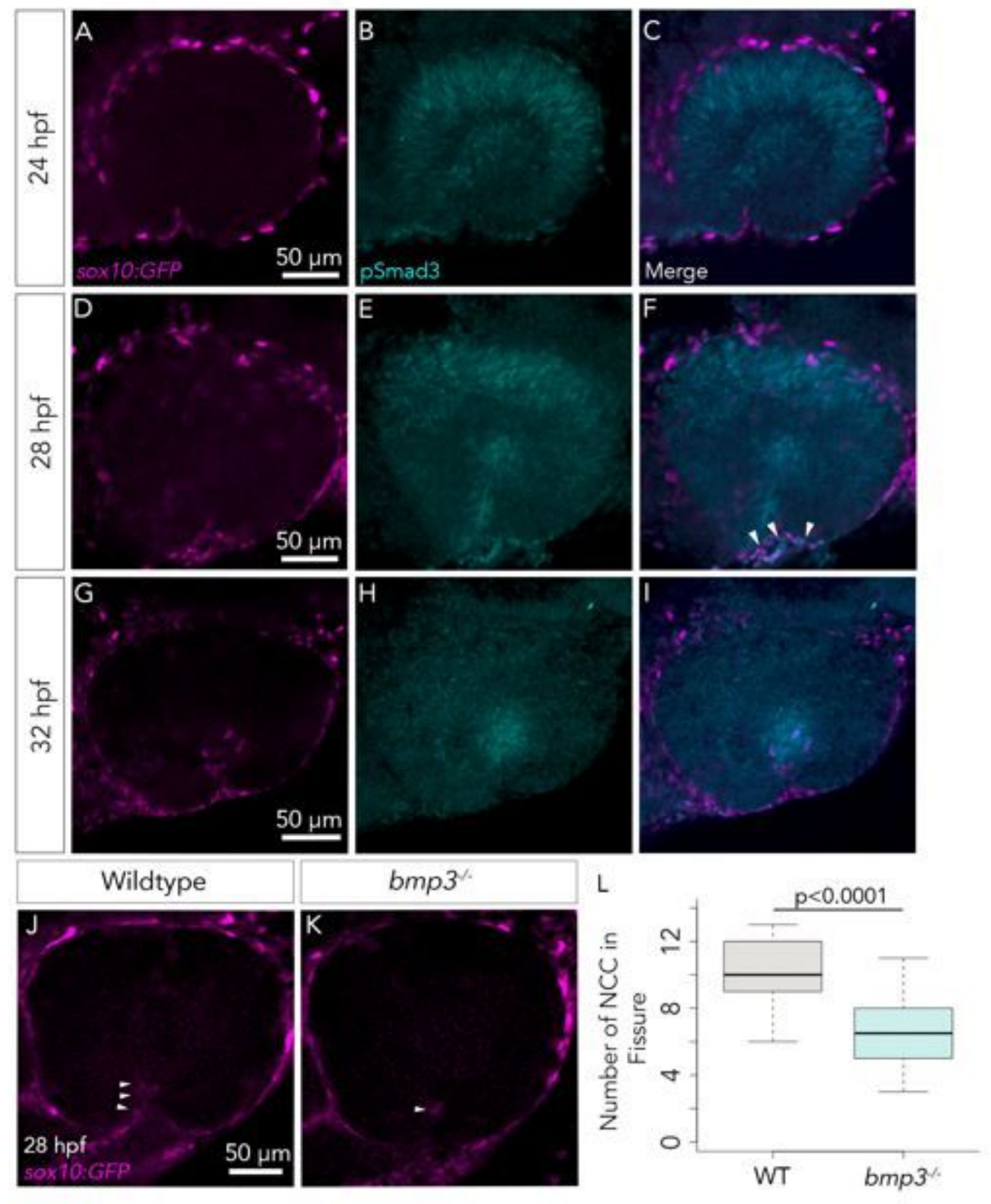

\section{Figure 7}

Bmp3 regulates periocular neural crest cells. A-I) pSmad3 immunofluorescence was conducted on wildtype $\mathrm{Tg}$ (sox10:EGFP) transgenic embryos at 24, 28, and $32 \mathrm{hpf}$. Almost no colocalization of neural crest cells with pSmad3 is seen at $24 \mathrm{hpf}(\mathrm{A}-\mathrm{C})$, but at $28 \mathrm{hpf}$ there are several neural crest cells in the ventral eye that colocalize strongly with pSmad3 (D-F, arrowheads). By $36 \mathrm{hpf}$ (G-I), the colocalization 
between pSmad3 and sox10+ neural crest cells in the ventral eye has diminished. J-L) To assess the effect of bmp3 on periocular neural crest cells, the number of sox $10+$ cells was examined in bmp3-/mutants (K) and wildtype siblings ( $\mathrm{J}$ ) on a Tg(sox10:EGFP) transgenic background at $28 \mathrm{hpf}$. L) There are significantly fewer neural crest cells in the choroid fissures of bmp3-/- embryos (6.56 \pm 1.04 cells, $n=18$ eyes) compared to wildtype siblings ( $10.1 \pm 1.00$ cells, $n=18$ eyes) (two sample t-test, $t 34=4.76$, p<0.0001). (WT=wildtype).

\section{Supplementary Files}

This is a list of supplementary files associated with this preprint. Click to download.

- Supplementallnformation.docx 